\title{
RNA polymerase redistribution and increased gene dosage support growth in $E$. coli strains with a minimal number of ribosomal RNA operons
}

Jun Fan a,b, , Hafez El Sayyed ${ }^{a, d}$, Oliver J. Pambos ${ }^{a, d}$, Mathew Stracy $^{\mathrm{a}, \mathrm{c}}$, Jingwen Kyropoulos ${ }^{\mathrm{a}}$, and Achillefs N. Kapanidis ${ }^{a, d, *}$

aBiological Physics Research Group, Clarendon Laboratory, Department of Physics, University of Oxford, Oxford OX1 3PU, United Kingdom

${ }^{\mathrm{b}}$ Institute of Fundamental and Frontier Sciences, University of Electronic Science and Technology of China, Chengdu, Sichuan, 611731, China

'Department of Biochemistry, University of Oxford, Oxford OX1 3QU, United Kingdom

${ }^{d}$ Kavli Institute for Nanoscience Discovery, New Biochemistry building, University of Oxford, South Parks Road, Oxford, OX1 3QU, United Kingdom

*To whom correspondence should be addressed. Tel: +44-1865-272-226; Email: kapanidis@physics.ox.ac.uk. Correspondence may also be addressed to Jun Fan. Tel: +86-2861-833-783 Email: fan.jun@uestc.edu.cn.

\section{ABSTRACT}

Bacterial transcription by RNA polymerase (RNAP) is spatially organised. RNAPs transcribing highly expressed genes locate in the nucleoid periphery, and form clusters in rich media, with several studies linking RNAP clustering and transcription of ribosomal RNA $(r r n)$. However, the nature of RNAP clusters and their association with $r r n$ transcription remains unclear. Here we address these questions by using single-molecule tracking to monitor the subcellular distribution of mobile and immobile RNAP in strains with a heavily reduced number of chromosomal $r r n$ operons ( $\Delta r r n$ strains). Strikingly, we find that the fraction of chromosome-associated RNAP (which is mainly engaged in transcription) is robust to deleting 5 or 6 of the 7 chromosomal $r r n$ operons. Spatial analysis in $\Delta r r n$ strains showed substantial RNAP redistribution during moderate growth, with clustering increasing at the cell end-caps, where the remaining $r r n$ operons reside. These results support a model where RNAPs in $\Delta r r n$ strains relocate to copies of the remaining $r r n$ operons. We also show that $\Delta r r n$ strains experience increased $r r n$ gene dosage in rich media, minimising growth defects due to $r$ deletions. Our study further links RNAP clusters and $r r n$ transcription, and offers insight on how bacteria maintain growth in the presence of only 1-2 rrn operons. 


\section{INTRODUCTION}

Transcription, a central process in gene expression, is spatially organised in many organisms; this organisation is thought to increase the efficiency for RNA synthesis (1) and help cells adapt to different growth environments, nutrients, and types of stress. In eukaryotes, synthesis of ribosomal RNA by RNA polymerase I occurs in the nucleolus, a nuclear compartment (2); further, eukaryotic mRNA transcription occurs in spatially enriched foci called "transcription factories" (3), which contain RNA polymerase II clusters (4) with lifetimes correlated to the levels of mRNA synthesis (5). Some viral transcription systems are also spatially organised; e.g., RNA polymerases of poliovirus form planar arrays/lattices with hundreds of molecules (6).

Transcription has also been shown to be spatially organised in bacteria, where early studies using conventional fluorescence microscopy in fixed cells showed that fluorescent derivatives of RNA polymerase (RNAP) in E. coli and B. subtilis (7) form bright, diffraction-limited foci in rich media, but not in minimal media; these prokaryotic transcription foci have been likened to transcription factories $(3,8)$. Subsequent studies using photo-activated localization microscopy (PALM), a super-resolution imaging method, provided further insight into RNAP spatial organisation; using PALM microscopy on fixed cells at different growth conditions, it was shown that RNAPs form large clusters with $\sim 70$ and $>100$ molecules in rich media, and smaller clusters with $\sim 35$ molecules in minimal media (9). Single-molecule localisation studies in live E. coli cells showed that RNAPs tend to co-localize with the nucleoid lobes, while being nearly absent from the ribosome-rich cell endcaps $(10,11)$. Further live-cell work combining PALM and single-molecule tracking was able to distinguish between mobile RNAPs (i.e., RNAPs exploring the nucleoid for promoters), and immobile RNAPs, with the latter fraction including transcriptionally active RNAPs that localized primarily at the nucleoid periphery; this study also provided the first observation of RNAP clustering in living bacteria (12).

Surprisingly, subsequent localisation-based work (13) suggested that RNAP clustering remained significant even when transcription was supressed, and only decreased substantially when all transcription was inhibited by rifampicin, leading to the proposal that the underlying nucleoid (rather than high transcription activity) controls the organisation of these RNAP clusters. Recently, it was suggested that RNAPs in bacteria form "biomolecular condensates" (14) via liquid-liquid phase separation (LLPS), a phenomenon seen in many organisms (15-17), including bacteria (18-21); the condensates were shown to contain high-density RNAP clusters in fast-growth conditions, and were mediated by protein-protein interactions, offering LLPS as an alternative mechanism that drives RNAP clustering (22).

A central point of debate in the spatial organisation of transcription and the formation of transcription foci is the exact role of ribosomal RNA operons ( $r r n$ ). Ribosomal RNA transcription (which involves 16S, $23 \mathrm{~S}$, and $5 \mathrm{~S}$ rRNA) accounts for $\sim 85 \%$ of all active transcription in fast-growing cells (23); such high transcription levels are essential for sustaining rapid synthesis of the $\sim 55,000$ ribosomes (10) needed per daughter per cell cycle during rapid growth (24). Notably, rrn transcription is much less prevalent in minimal media. In the genomic map of $E$. coli, most of the seven rrn operons locate near the origin of replication (oriC), and all $r r n$ operons orient in the same direction as DNA replication (Fig. 1A); this chromosomal location leads to increased gene dosage for $r$ rn genes. 
Transcription foci and RNAP clustering have been linked to $r r n$ operons even during the first RNAP distribution studies $(1,9)$, which raised the possibility that transcription foci involve multiple (perhaps all) rrn operons operating in close proximity and in a growth-dependent manner (since such foci were absent in minimal media), supporting a "bacterial nucleolus" model $(2,24)$. Consistent with that model, Gaal et al measured the pairwise distance of $r r n$ operons in single cells and found that 6 out of the $7 \mathrm{rrn}$ operons in E. coli are in close proximity in 3D space (Fig. 1A, and Ref. (25)). Endesfelder et al also linked RNAP clustering to rrn operons, and suggested that clusters with 35-70 molecules represent single $r r n$ operons, and large clusters (>100 molecules; $50-300 \mathrm{~nm}$ in diameter) represent superclustered multiple rrn operons (9). These indirect links were supported by Weng et al (13), which directly showed that RNAP clusters were indeed colocalising with sites of high rrn transcription in rich media, while the formation of these clusters was independent of $r r n$ transcription activity (13). The persistence of significant clustering despite the dramatic loss in rrn transcription was later attributed to LLPS (22).

Despite the progress in the understanding of spatial organisation of transcription, there are still many open questions. What is the link between RNAPs clusters and $r n$ operons, if any, in minimal media? To what extent LLPS contributes to RNAP clustering forming on rrn operons? What are the mechanisms that maintain the ability of cells to grow even when the number of chromosomal $r r n$ operons is very small (1-2 copies)?

Here, we study the link between RNAP clusters and $r r n$ operons by using single-molecule imaging and tracking $(26,27)$ to obtain the RNAP spatial distribution and mobility in strains featuring deletions of most rrn operons ( $\Delta r r n$ strains; Fig. 1B). We show that, remarkably, in strains with only one or two chromosomal $r$ copies, bacterial cells maintain the same level $(\sim 48 \%)$ of immobile RNAP (which mainly reflects RNAP engage in transcription) during moderate growth rates; immobile RNAPs in $\Delta r r n$ strains move close to cell endcaps, suggesting that RNAPs relocate to the remaining $r$ rn operons, which have a pole-proximal location. During fast growth in rich media, loss of most $r r n$ operons decrease slightly the immobile RNAP fraction; for the strains with one and two remaining rrn operons on the chromosome, we show the presence of increased replication initiation in rich media, which will further increase the gene dosage for the remaining $r r n$ genes. RNAPs retained its clustering in the $\Delta r r n$ strains, whereas colocalization analysis showed a good correlation between RNAP clusters and $r$ rn operons. In general, our results establish that loss of $r$ operons is compensated through increased RNAP occupancy and increased gene dosage of the remaining chromosomal $r r n$ operons. Our work expands our understanding of how RNAP is organised and allocated between transcription activities and how bacteria regulate their transcription to adapt to variations of their chromosomal content and growth environment.

\section{MATERIALS AND METHODS}

Bacterial Strains. The rpoC:PAmCherry WT strain carrying PAmCherry fused to the $\beta$ ' subunit under the control of its native promoter used was built as described previously (9). The $\Delta r r n$ strains were obtained by P1-transduction of the rpoC:PAmCherry gene in the $\Delta r r n$ strains. The deletion strains were acquired from CGSC ( $E$. coli Genetic Stock Center at Yale): SQ88 as $\Delta 5 \mathrm{rrn}$ with $r \mathrm{rnB}$ and $r r n C$ remaining, SQ110 as $\Delta 6 \mathrm{rrn}$ with $r \mathrm{rnE}$ remaining and SQ2158 as $\Delta 7 \mathrm{rrn}$ supplemented with plasmidborne $r r n B$ (pK4-16, based on pSC101, see also Ref (28)). For gene-dosage studies, parS ${ }^{\text {MMT1 }}$ sites 
(29) were constructed by $\lambda$ red recombination (30) around the $r n C$ or $r r n E$ at the precise genomic positions used in (25). The parS-containing operons were introduced in rpoC:PAmCherry WT, $\triangle 5$, and $\Delta 6$ strains via P1 transduction. Foci visualization was obtained by constitutively expressing ParB-yGFP that selectively binds to parS sites from the pFH2973 (29); the strains used for this experiment were simply denoted by a parS suffix.

Cell growth rate measurements were performed using OD600 on a microplate reader (FLUOStar, BMG Labtech). Three separate measurements were carried out with individual blank media. The absorbance of OD600 was measured every 5 min for 16 hrs to generate the growth curves.

Cell preparation for imaging. Strains were streaked onto LB plates supplemented with required antibiotics for each strain. For WT, we used $100 \mu \mathrm{g} / \mathrm{ml}$ ampicillin; for $\Delta 5$ and $\Delta 6$, we used $100 \mu \mathrm{g} / \mathrm{ml}$ ampicillin and $40 \mu \mathrm{g} / \mathrm{ml}$ spectinomycin, respectively; and for $\Delta 7$, we used $100 \mu \mathrm{g} / \mathrm{ml}$ ampicillin, $40 \mu \mathrm{g} / \mathrm{ml}$ spectinomycin and $50 \mu \mathrm{g} / \mathrm{ml}$ kanamycin. Single colonies were inoculated into LB and grown at $37^{\circ} \mathrm{C}$ and $220 \mathrm{rpm}$ for a pre-culture of $2 \mathrm{~h}$, then diluted 1/250 into M9Glu media ( 1 X M9 media supplemented with $\mathrm{CaCl}_{2}, \mathrm{MgSO}_{4}$ and $0.2 \%$ glucose but without any additional vitamins or amino acids) or RDM (rich defined medium; Teknova) and grown at $37^{\circ} \mathrm{C}$ for overnight. Overnight cultures were diluted into fresh media and grown for $>2 \mathrm{~h}$ at $37^{\circ} \mathrm{C}$ till early exponential phase (OD 0.1-0.2 for M9Glu culture, or OD 0.2 for RDM culture). $1.5 \mathrm{ml}$ cell culture was centrifuged down, concentrated into $30 \mu \mathrm{l}$, and immobilized on $1 \%$ low-fluorescence agarose (BioRad) pads (supplemented with required M9Glu or RDM to keep media consistent). After immobilising the cells on agarose pads with fresh media, we monitored the RNAP localization using single-particle tracking on a PALM microscope and measured the apparent diffusion coefficient $\left(D^{*}\right)$ of RNAPs (see also Fig. 1C-D).

For fixed-cell colocalization experiments combining PALM with FISH, $1.5 \mathrm{ml}$ of culture of the WT or $\Delta r r n$ strains carrying the rpoC:PAmCherry were spun down and then resuspended into $1 \mathrm{ml}$ of PBS. One $\mathrm{ml}$ of $4 \%$ PFA was mixed $1: 1$ with the bacterial culture and incubated for 40 min with mild shaking on a nutator mixer at RT. After 3 washes with PBS, we added $500 \mu \mathrm{l}$ of absolute ethanol to permeabilize the cells, and washed twice with PBS. We then immobilized $20 \mu$ l of cells on chitosan (31) housed in a selfadhesive gasket. For the FISH studies, $5 \mu \mathrm{M}$ of the pre-rRNA probes carrying the sequence [Atto488]TGCCCACACAGATTGTCTGATAAATTGTTAAA-GAGCAGTGCCGCTTCGCT (13) was incubated with the permeabilized cells and incubated for $5 \mathrm{~min}$ at room temperature, then washed $3 \mathrm{x}$ with PBS. Cell were then imaged as discussed below.

Single-molecule imaging of living cells. A custom-built single-molecule tracking PALM microscope (12) was used for the imaging of single RNAP-PAmCherry molecules and the detection of diffractionlimited $r r n$ foci. Cells mounted on $1 \%$ agarose pads were imaged under bright-field illumination to perform cell segmentation. Prior to PALM imaging, pre-activated PAmCherry molecules were photobleached under continuous 561-nm excitation. Sparse photoactivation of the remaining population of PAmCherry was performed by continuous exposure to low intensity $405-\mathrm{nm}$ excitation, such that the dataset consists of well separated single molecules. Under simultaneous excitation with a 561 -nm laser, these photoactivated molecules fluoresce until permanently photobleached. Imaging was performed at a frame rate of $15 \mathrm{~ms} /$ frame for at least 30,000 frames, until the entire pool of RNAP-PAmCherry molecules had been imaged. 
Imaging and counting specific rrn operons by counting parB foci in live cells. Using parScontaining operons in strains $\mathrm{WT}, \Delta 5$, and $\Delta 6$ supplemented with a plasmid constitutively expressing ParB-yGFP (see Bacterial Strains), we obtained estimates of the copy number of specific rrn operons in the WT and deletion strains. Cells were grown in RDM until OD600 reaches 0.2, immobilized on agarose pads, and imaged using 473-nm laser excitation and 500-ms exposures. The distribution of the number of foci per cell for the different strains was obtained after segmentation and localisation of the bright fluorescence parB foci using microbe, a plug-in for ImageJ (32). The results were then exported and plotted as a frequency histogram of the number of foci per cell.

Two-colour colocalization assay. For FISH imaging of $r r n$ foci with fixed cells, a 488-nm laser was used for 20 frames at 500-ms exposures. Bright field and pre-bleaching with a 561-nm laser were performed as for live cells. Excitation of RNAP-PAmCherry molecules was performed using a 561-nm laser for 90,000 frames at $15 \mathrm{~ms} /$ frame to capture the entire pool of RNAP molecules.

Image processing and data analysis. Live-cell data were processed following published procedures based on a custom-written MATLAB software (12) for localizing single RNAP-PAmCherry molecules and tracking within a region-of-interest to distinguish different species. Histograms of apparent diffusion coefficient were fitted as two-gamma distributions, with the value for the immobile species fixed at 0.09 $\mu \mathrm{m}^{2} / \mathrm{s}$. Comparison of different strains in defined growth media was done by collecting data in triplicates. Fixed cell data were processed using rapidSTORM as previously $(9,33)$ to localize single RNAPPAmCherry molecules while removing any repeated localisations in flanking frames.

Clustering analysis of RNAP localisations. Clustering analysis of RNAP molecules localized by rapidSTORM was done using a MATLAB implementation of the DBSCAN algorithm (see Refs $(9,34)$ and also Yarpiz page: Mostapha Kalami Heris, DBSCAN Clustering in MATLAB (URL: https://yarpiz.com/255/ypml110-dbscan-clustering), Yarpiz, 2015). From a Monte-Carlo simulation of localisations of RNAPs in M9Glu media and based on previous measurements in fixed cells (9), we determined the appropriate parameters for reliable clustering to be $\varepsilon=20 \mathrm{~nm}$ and MinPts $=4$.

Pair correlation of RNAP localisations in live cells was performed as described previously (12). Briefly, the pair correlation function, $g(r)$ was computed for all pairwise distances between the first localisations of each trajectory. For each cell, an equal number of simulated molecules were then distributed uniformly throughout the same segmented cell volume to generate another $g(r)$ function which was used to normalise the experimental result. This normalisation compensates for the finite and asymmetric geometry of the cell.

Pair correlation of RNAP clusters with $r r n$ foci. Analysis of $r r n$ foci in fixed cells was performed by bandpass filtering, followed by free elliptical Gaussian fitting for localisation. The pair correlation $\mathrm{g}(\mathrm{r})$ function was computed for all RNAP-rrn distances within the cell. A uniform distribution of the same number of molecules was generated throughout the segmented nucleoid area and used to normalise the experimental data. According to (35), nucleoid area covers $\sim 56 \%$ of the overall cell area in M9Glu media, therefore we shrink both the cell length and width to $\sim 75 \%$ to estimate the nucleoid area. A second uniform distribution was then generated and normalised using the same distribution as the experimental data to provide a visual guide for completely uncorrelated data, shown as the dashed line 
in the plots of the pair correlation function. Finally, the fraction of molecules found within $200 \mathrm{~nm}$ of $r \mathrm{rn}$ foci was calculated for both the experimental and uniform data.

Heatmap plotting. Heatmaps of RNAP distributions were generated by pooling large populations of cells into two groups by cell length. Thresholds for cell length were selected such that the groups are composed of cells containing single and double nucleoids (Fig. S2). 2D histogram data of molecule location were obtained by binning data from normalised cell coordinates defined by the microbeTracker segmentation of each cell. Normalised data from all cells within the same cell length group were combined to produce heatmaps for mobile and immobile molecules. A difference map was generated by subtracting the mobile from the immobile distributions. Heatmaps were projected along their long and short axes to illustrate the RNAP distribution throughout the cell volume.

Simulations. 3D simulations of RNAP molecule locations were performed using Monte Carlo methods for the wild type, $\Delta 5$, and $\Delta 6$ strains. RNAP molecules were categorised into four populations; mobile, bound to $r$ rn operons at the nucleoid periphery, bound in small clusters throughout the nucleoid, and 'noise' found throughout the cell volume. A total of 1800, 1800, and 1200 molecules were distributed between these categories in wild type, $\Delta 5$, and $\Delta 6$ respectively, consistent with experimental results in Fig. S9. A split of $48 \%$ immobile, $52 \%$ mobile molecules was simulated based on experimental data presented in Fig. 1.

A probability density function for non-rrn associated small RNAP clusters was obtained by fitting cluster size distributions obtained experimentally to an exponential function of the form $y=a e^{-b x}$. The simulated population of non-rrn small cluster sizes are then generated by sampling from the inverse transform of this exponential probability density function, $X=-(1 / b) \ln (1-(b u / a))$, where $u[0: 1]$ is a set of uniformly distributed random numbers. The centres of non-rrn associated RNAP clusters were distributed uniformly throughout the nucleoid volume, around which RNAP molecules were distributed isotropically with a Gaussian radial density profile. Any molecules generated outside of the modelled cell volume were regenerated until a complete distribution was obtained.

The mobile population distributed uniformly within the nucleoid was generated via rejection sampling within a prolate spheroid volume positioned with a 150-nm separation between the nucleoid and cell poles. The same code was used to position the centres of small (non-rrn) clusters. A fraction of the mobile population was diverted to a population of localisation 'noise' that was generated uniformly across the entire cell volume via rejection sampling.

rrn operons were positioned along the pole-proximal periphery of each nucleoid. Simulations were performed with 7 operons/nucleoid in wild type, 2 in $\Delta 5$, and 1 in $\Delta 6$. The location of each cluster centre along the long axis which was weighted by the genomic distance of each $r \mathrm{rn}$ operon relative to oriC, defining a ring of possible locations around the nucleoid periphery. Candidate locations around this ring were proposed until a position was obtained with a minimum separation of at least $70 \mathrm{~nm}$ from other $\mathrm{rrn}$ operons. RNAP molecules were then distributed around each of these rrn cluster centres isotropically as described above for small non-rrn clusters. The proportion of immobile molecules associated with rrn operons was simulated across the range of 30 to $80 \%$, with $60 \%$ to most closely matching the experimental data shown in Fig. 4. These 3D simulations were then projected into $2 \mathrm{D}$, and analysed by 
computing the pair-correlation function $g(r)$ for each cell. The process was then automated for 2000 cells to obtain the mean $g(r)$ for the distribution.

Estimation of the copy number of $r$ rn operons and rRNAPs for a given growth rate. The total number of RNAPs engaged with the $r$ operons $\left(\mathrm{N}_{r}\right)$ for each strain was estimated by interpolation of the $\mathrm{N}_{r}$ values from Ref (36) [which were calculated using the expression $\mathrm{N}_{r}=\mathrm{rr}_{r} / \mathrm{C}_{r}$, where $\mathrm{r}_{r}$ and $\mathrm{Cr}_{r}$ are the overall rate of rRNA synthesis and the rRNA elongation speed $(85 \mathrm{nt} / \mathrm{s})$ as measured by Bremer and Dennis (37)] by fitting to a single exponential. The expected number of $r r n$ operons in all strains for a given growth rate was obtained as in Bremer and Dennis (equation 9 in Table 5 of Ref (37)), by considering the growth rate of each strain, and the location of each $r r n$ on map of the $E$. coli chromosome.

\section{RESULTS}

\section{RNAPs remain heavily engaged with the chromosome despite deletion of most $r$ rn operons}

To clarify the relationship between RNAP clusters and $r$ rn operons, we compared a well characterised E. coli strain carrying all 7 chromosomal $r r n$ operons ("wild type", WT) with strains carrying a drastically reduced number of $r r n$ operons; these $r r n$ deletion strains $(\Delta r r n)$ were originally developed to study the link between $r r n$ operon multiplicity and ribosome function $(38,39)$.

Specifically, we studied a strain in which 5 out of 7 operons were deleted, leaving only $r r n B$ and $r r n C$ on the chromosome ( $\Delta 5$, Fig. 1B); a strain in which 6 out of 7 operons were deleted, leaving only $r r n E$ on the chromosome ( $\Delta 6$, Fig. $1 \mathrm{~B}$ ); and a strain in which all 7 chromosomal rrn operons were deleted, and instead supplemented by a low copy-number plasmid ( $\sim 5$ copies per chromosome) containing a single $r m B$ operon $(\Delta 7$, Fig. 1B). To enable tracking of single RNAP molecules in cells, all strains contained a fully functional $C$-terminal fusion of the $\beta$ ' subunit of RNAP with a photoactivatable mCherry (PAmCherry; in Refs $(9,12)$; see also Methods).

To check the fitness of $\Delta r r n$ strains relative to the WT, we monitored their growth in different media (Figs. 1B, S6); in general, the growth rates in the deletion strains correlated to the number of remaining copies of $r$ rn operons, with $\Delta 6$ being the slowest growing. The WT strain displayed a 47-min doubling time at $37^{\circ} \mathrm{C}$ in minimal M9 media supplemented with $0.2 \%$ glucose (M9Glu; see Methods); in comparison, $\Delta 7$ grew marginally more slowly (50 min), $\Delta 5$ grew significantly more slowly (57 min), and $\Delta 6$ grew substantially more slowly $(73 \mathrm{~min}$ ). The growth rates of $\Delta r r n$ strains in rich media followed a similar pattern; in rich defined medium (RDM), WT was the fastest growing (36 min), followed by $\Delta 7$, $\Delta 5$ and $\Delta 6$ (40, 46 and $51 \mathrm{~min}$, respectively; for LB, see Fig. S6). In general, reduction in the number of $r r n$ copies led to small-to-moderate decrease in the growth rate, presumably by affecting the rate at which different strains produce ribosomes $(24,40)$.

To follow the RNAP mobility in live cells, we performed single-particle tracking of RNAP molecules using photoactivated localisation microscopy (PALM) on surface-immobilised cells, as described (Ref (12); see also Methods). The single-molecule tracks allowed us to calculate apparent diffusion coefficients $\left(D^{*}\right)$ for hundreds of RNAPs per cell (Fig. 1C-E; see also Methods) and construct $D^{*}$ histograms. The $D^{*}$ distribution for RNAP in WT cells grown in M9Glu was described well by two RNAP fractions with 
different mobility: a species $\left(48.2 \%\right.$ of all tracks) that remains immobile ( $D^{*}$ of $\sim 0.09 \mu \mathrm{m}^{2} / \mathrm{s}$ ), and a mobile species ( $51.8 \%$ of all tracks) with $D^{*}$ of $0.36 \mu \mathrm{m}^{2} / \mathrm{s}$ (Fig. 1D, top; Fig. S1A, top). The immobile fraction includes RNAPs bound to the bacterial chromosome for several frames (12), which in turn correspond mainly to RNAPs bound to promoters and transcribed genes; the immobile species includes any RNAPs found in condensates, since they have been suggested to possess very low mobility (22). On the other hand, the mobile fraction corresponds to RNAPs interacting non-specifically and transiently with the entire chromosome during their promoter search (41).

To assess the effect of $r r n$ operon loss on RNAP mobility in M9Glu, we compared the $D^{*}$ distribution of WT with those of $\Delta r r n$ strains (Figs. 1D, 1F; Fig. S1C). In $\Delta 5$, and despite the deletion of 5 out of 7 chromosomal $r r n$ operons, the $\mathrm{D}^{*}$ distribution and the immobile RNAP fraction were essentially identical to those in WT (47.6\%; Fig. 1D, bottom; Fig. 1F). The lack of any significant mobility difference compared to WT was also observed for both $\Delta 6$ and $\Delta 7$ strains ( $48.8 \%$ and $46.4 \%$, respectively; Fig. $1 \mathrm{~F} \&$ Fig. S1B-C, left). These results establish that, despite the loss of most $r r n$ operons, $\Delta r r n$ strains show the same level of immobile RNAP as the WT.

To assess the effect of $r r n$ operon loss on RNAP mobility in rich media (RDM), where the $r$ rn operons should be much more heavily occupied by RNAPs than mRNA-coding genes (and where any RNAP condensates should be more visible, potentially accounting for $\sim 30 \%$ of the immobile fraction; Ref. (22)), we performed similar RNAP mobility comparisons between WT and $\Delta r r n$ strains in RDM. As we observed before (12), the immobile RNAP fraction in WT was $\sim 63 \%$ (Fig. 1E, top; Fig. 1G), while $\Delta 5$ showed a $\sim 6 \%$ decrease in the immobile fraction (57.3\%; Fig. 1E, bottom; Fig. 1G, Fig. S1C, right); the results for $\Delta 6$ and $\Delta 7$ showed a similar decrease in immobile RNAPs ( $58.3 \%$ and $57.5 \%$; Fig. 1G, Fig. S1B-C, right). In general, for all $\Delta r r n$ strains grown in rich media, the immobile RNAP fraction stays surprisingly at the same high level (57-58\%).

\section{DNA-bound RNAPs relocate to pole-proximal positions in $\Delta r r n$ strains in M9Glu}

To examine whether the deletion of most or all chromosomal $r$ rn operons leads to any RNAP relocation within cells, and to gain insight regarding any redistribution between cellular RNAP pools, we examined the spatial distributions of mobile and immobile RNAPs in $\triangle r r n$ strains via sorting single-molecule tracks using a $D^{*}$ threshold (Fig. 2A-B; see also Ref. (12) and Methods). To capture the average behavior for cells of similar size, we pooled the normalized positions of RNAPs from individual cells within different size ranges, and generated spatial heat-maps for both mobile and immobile fractions (see Methods).

As we observed previously (12), the RNAP spatial distribution in WT cells with two nucleoids in M9Glu showed that mobile RNAPs localise throughout the nucleoid, essentially highlighting the nucleoid location (Fig. 2B, left), whereas immobile RNAPs tended to localize at the nucleoid periphery (Fig. 2B, middle); this redistribution towards the periphery for immobile molecules can be seen more clearly in the normalised difference heatmap between the two mobility fractions (Fig. 2B, right). Similar results were obtained for shorter cells, which carry only a single nucleoid (Fig. S2).

We then examined strain $\Delta 5$ to see how the deletion of $5 \mathrm{rrn}$ operons affects the RNAP spatial distribution. The mobile RNAPs in $\Delta 5$ had a spatial distribution nearly identical to the WT one, i.e., spanning the entire nucleoid (Fig. 2C, left), reflecting the transient, non-specific interactions of this 
target-searching RNAP fraction with the nucleoid (41). In contrast, the spatial distribution of immobile RNAPs in $\Delta 5$ is substantially different to the WT one, with immobile RNAPs becoming much more concentrated at the pole-proximal edges of nucleoid (Fig. 2C, middle; see also the difference heatmap, Fig. $2 \mathrm{C}$, right). For a clearer view of this RNAP relocation, we projected the heatmaps along cell length (Fig. 2E-F). While the projection for mobile RNAPs is similar, the projection of immobile RNAPs shifts from a fairly flat distribution centred at $\sim 30 \%$ of cell length for WT, to a distribution with a peak at $\sim 15 \%$ of cell length and $\Delta 5$ (green line, Fig. $2 \mathrm{~F}$ ); this shift is also reflected by the large increase in the RNAP fraction localised in the exterior $25 \%$ region along the long axis (53\% in $\Delta 5$ vs. $42 \%$ in wild type; Fig. $2 \mathrm{G})$; this increase corresponds to the relocation of $\sim 10 \%$ of all immobile RNAPs.

A profile similar to $\Delta 5$ was observed for $\Delta 7$, which features only plasmid-borne $r r n$ operons: the mobile RNAPs cover the entire nucleoid (Fig. 2D, left), while $\sim 10 \%$ of the immobile RNAPs relocate to poleproximal regions (Fig. 2D, middle and right; Fig. 2G), shifting the peak in the projection of immobile RNAPs to $\sim 15 \%$ of cell length (Fig. $2 \mathrm{~F}$, red line). The $\Delta 6$ strain (with a single chromosomal $r$ rn operon) also behaved similarly to $\Delta 5$ and $\Delta 7$ for both mobile and immobile RNAPs (Fig. 2E-G), with the main differences being the position of the new peak of immobile fraction, which appears at $\sim 20 \%$ of cell length (Fig. 2E, blue line). Similar results were obtained for shorter cells (Fig. S3).

To explain our spatial distributions of immobile RNAPs, we need to consider that they contain several RNAP pools: RNAPs transcribing $r r n$ operons (rRNAPs), RNAPs transcribing mRNAs (mRNAPs), and any condensate-associated RNAPs (cRNAPs). Removal of several rrn operons from the chromosome essentially releases many rRNAPs; since the immobile fraction for all $3 \Delta r r n$ strains does not change relative to WT, the released rRNAPs must join one or more of the 3 main pools of immobile RNAPs. Since the $\Delta r r n$ strains do not have a substantial growth defect, it is likely that to maintain sufficient rRNA synthesis to support ribosome biogenesis, many (perhaps all) of the released rRNAPs are captured by the remaining $r r n$ operons. Our results also show that a large fraction of immobile RNAPs in the $\Delta r r n$ strains engage with the entire nucleoid (Fig. 2C-D middle); we attribute this fraction mainly to non-rrnassociated immobile RNAPs.

\section{Immobile RNAPs spread throughout the nucleoid in rich media}

Since growth conditions influence dramatically the RNAP spatial distribution (1), we examined the spatial distribution in $\Delta r r n$ strains growing exponentially in rich media (Fig. 3), a condition wherein cells need to accumulate high numbers of ribosomes (up to 70,000; see also Ref. (39)) and thus require high rrn expression $(42,43)$. In rich media (RDM), WT cells divided every $\sim 36 \mathrm{~min}$, a growth rate that corresponds to $\sim 2.7$ chromosomes/cell on average, and a high copy of $\mathrm{rrn}$ genes ( $\sim 23 \mathrm{rrn} / \mathrm{cell}$; Refs $(36,37))$. The RNAP distribution in the WT strain in RDM showed that, as in M9Glu, mobile RNAPs explore the entire nucleoid, whereas many immobile RNAPs appear in clusters distributed throughout the nucleoid (see next section), with some enrichment at the nucleoid periphery (Fig. 3A; Fig. 3B; Fig. S4); this enrichment, especially visible along the short cell axis, is clear in the difference map between immobile and mobile populations, both for long and short cells (Fig. 3B, right; Fig. S4).

In contrast to its profile in M9Glu, the $\Delta 5$ strain shows a profile similar to WT for both mobile and immobile RNAPs, i.e., both populations are evenly distributed along the long axis of cells, and much of 
the immobile population appears clustered in single cells (Fig. 3A, $2^{\text {nd }}$ row). Intriguingly, and in contrast to the profile in M9Glu, no apparent relocation of immobile RNAPs to the poles is observed (Fig. 3C; Fig. S4), with the RNAP fraction in the pole-proximal region along the long axis being essentially identical for $\Delta 5$ and WT, both for mobile and immobile RNAPs (Fig. 3E-F). Broadly similar profiles and observations were seen in $\Delta 7$ (Fig. 3A, bottom; Fig. 3D) and $\Delta 6$ (Fig. S4; Fig. 3E-F), as well as in populations of short cells (Fig. S5). Notably, long cells feature a more polar and "nucleoid excluded" localisation of immobile RNAPs in $\Delta 7$ relative to the mobile population (Fig. 3D), likely reflecting the localisation of most plasmids.

To explain the spatial distributions of immobile RNAPs, we consider that, during fast growth conditions in rich media, cells contain multiple copies of the chromosome $(9,44)$ and multiple sets of $r r n$ operons, with the ori-proximal location of $r r n$ genes further increasing the number of $r r n$ copies (45); for example, for $\Delta 5$, we expect the group of long cells to have $\sim 3$ chromosomes and $\sim 8 \mathrm{rrn}$ copies on average (37). As in M9Glu, removal of several $r$ operons from the chromosome releases many rRNAPs; since the bound fraction for all $3 \Delta r r n$ strains in RDM decreases only by $\sim 6 \%$ relative to $\mathrm{WT}$, the released rRNAPs must join one or more of the 3 main pools (rRNAPs, mRNAPs, and cRNAPs). We reason that, to maintain sufficient rRNA synthesis to support ribosome biogenesis (albeit at reduced growth rates), most released rRNAPs in $\Delta r r n$ strains are re-captured by the remaining $r r n$ operons (see also Discussion).

Our interpretation above is consistent with the location of RNAP clusters in single cells (e.g., $\Delta 5$ cells in Fig. 3A), which roughly map to the expected location of the 4 replication origins for cells of this size and growth rate (notably, the remaining $r r n$ operons in $\Delta 5$ are proximal to ori). However, since the group of long cells covers a range of lengths (3.5-4.5 $\mu \mathrm{m})$, and since the location of $r r n$ operons varies for cells of different length, the average picture for the group of long cells is blurred and features fairly continuous distributions that do not reflect the localised nature of the clusters seen in single cells. On the other hand, $\Delta 7$ shows a clear profile of nucleoid exclusion for a large fraction of immobile RNAPs, suggesting that these represent RNAPs transcribing $r$ rn genes on nucleoid-excluded plasmids. These rrn-centred RNAP pools are in addition to any pools of cRNAPs, although it is unclear whether cRNAP pools nucleate on the rrn-centred RNAP pools, or exist in isolation.

\section{RNAP clustering increases upon loss of most chromosomal rrn operons}

The RNAP spatial distribution in $\Delta r r n$ established that RNAPs in M9Glu relocate in pole-proximal regions, raising the possibility that relocation forms new clusters or enlarges smaller ones. To assess the level of RNAP clustering, we performed clustering analysis using the DBSCAN algorithm (Ref. (34) and Methods). Analysis of WT grown in M9Glu and subsequently fixed (Fig. 4A, top) showed that $29 \%$, $11 \%$, and $9 \%$ of clustered RNAPs were found in clusters with $>35,>70$, and $>100$ molecules, respectively ( $C>35, C>70$, and $C>100$ species, Fig. 4B top) (9). For the $\Delta 5$ strain in M9Glu media, which shows an RNAP copy number per cell similar to that of WT (1767 vs 1844; Fig. S9), we detected a significant increase in all three species of large clusters (Fig. 4B), and notably for the $C>70$ population ( $17 \%$ for $\Delta 5$ vs $11 \%$ for $W T$ ), suggesting that RNAP redistribution increases the abundance of large clusters. Similar results were observed for $\Delta 7$, where despite a lower measured RNAP copy number for $\Delta 7$ (1285 vs 1844 for WT), the C>70 population increases to $20 \%$ (cf. $11 \%$ for WT; Fig. 4B, bottom). 
Interestingly, there is a small reduction in large clusters in $\Delta 6$, in part due to a decrease in the copy number of RNAPs (1140; Fig. S9). These results are consistent with the deletion of most $r r n$ increasing the degree of clustered RNAP due to relocation of RNAPs to the remaining rrn copies, all found in poleproximal regions.

To gain another perspective to RNAP clustering, we performed pair-correlation analysis of the RNAP localisations (12), wherein the distances between all pairs of individual molecules are analysed and compared to a random distribution; a pair-correlation $g(r)$ value of $>>1$ for a range of inter-molecular distances indicates significant clustering, whereas $g(r) \sim 1$ indicates a non-clustered distribution. Notably, the pair-correlation analysis is unaffected by differences in RNAP copy numbers per strain, and requires no optimisation in analysis parameters. We first examined RNAPs in fixed cells grown in M9Glu, and observed that RNAPs in WT show only slight clustering at distances within $\sim 100 \mathrm{~nm}$, whereas all three $\Delta r r n$ strains showed much higher clustering within the $\sim 100 \mathrm{~nm}$ range (Fig. $4 \mathrm{C}$ ), with $\Delta 7$ being the most clustered strain. Pair-correlation analysis on simulated data for the two strains retaining chromosomal rrn copies ( $\Delta 5$ and $\Delta 6$; see Methods) also showed that WT is expected to maintain the lowest level of RNAP clustering relative to $\Delta 5$ and $\Delta 6$ (Fig. 4D), consistent with our experimental results.

We also performed pair-correlation analysis in live cells in M9Glu. These experiments are complicated by any 3D motions of clustered RNAPs during the $\sim 8 \mathrm{~min}$ of imaging; such motions will reduce the pair correlation and spread it out to longer length-scales; however, any persistent clustering should still be visible. We first analysed all RNAP localisations from the entire acquisition, and observed that all $\Delta r r n$ strains show increased RNAP clustering relative to WT, with $\Delta 7$ and $\Delta 6$ being the most clustered (Fig. $4 \mathrm{E})$; relative to fixed cells, the distance over which clustering is observed increases for all strains, as expected from the presence of some cluster mobility.

We then performed pair-correlation analysis on the mobile and immobile RNAP species in live cells in M9Glu; since we do not anticipate mobile RNAPs to be clustered (apart from exploring the entire nucleoid; as such, they do not fill the entire cell), this analysis should offer clearer views of the clustering of immobile RNAPs. Indeed, mobile RNAPs for all strains do not cluster (Fig. 4F, dotted lines); in contrast, the immobile RNAPs of both WT and $\Delta r r n$ strains (Fig. 4F, solid lines) appear much more clustered than mobile RNAPs. Further, all strains show a similar level of clustering for immobile RNAPs. Taken together, our results indicate that in M9Glu, RNAPs become more clustered, consistent with the remaining $r r n$ operons in $\Delta r r n$ strains accommodating relocated RNAPs to compensate the loss of many chromosomal rrn operons.

In rich media, RNAPs in fixed cells of WT are more clustered than in M9Glu, whereas, in contrast, RNAPs in all $\Delta r r n$ strains show reduced clustering relative to their levels in M9Glu, and relative to the WT in RDM (Fig. 4G). Pair-correlation analysis on the immobile RNAP molecules in live cells show similar differences between WT and the $\Delta r r n$ strains (Fig. $4 \mathrm{H}$ ); further, the levels of clustering in all strains exceed significantly the clustering seen in M9Glu. This result reinforces our qualitative observations of clustering in the discussion of the spatial RNAP distribution in RDM; the absence of prominent peaks in the projection of immobile RNAP localisations in the $\Delta r r n$ strains are not due to the presence of highly distributed immobile RNAPs, but rather due to the presence of RNAP clusters with 
variable positions along the long-cell axis (due to the lack of synchronisation of the cells, and, in turn, due to variable positions of the remaining $r r n$ operons).

\section{The expected cellular $r r n$ copy number in $\Delta r r n$ strains can sustain the measured growth rates in M9Glu, but not in RDM}

To evaluate whether the remaining $r$ rn copies can accommodate the number of RNAPs needed to sustain the measured growth rate (which is proportional to both cellular ribosome content [hence to the rRNA cellular content] and peptide elongation rate (46)), we estimated the copy number of $r$ rn operons (37) and of rrn-associated RNAPs (36) for the growth rates of our strains (Table 1), and used them to estimate the average fractional occupancy of each $r r n$ operon (Table 1).

For the WT strain (doubling time $\mathrm{T}$ of $\sim 47 \mathrm{~min}$ in M9Glu), we expect an average of $\sim 2$ genome equivalents and $\sim 17.5 \mathrm{rrn}$ operons per cell (43), and $\sim 400$ rRNAPs (38), yielding an average of $\sim 23$ RNAPs $/ r r n$. Considering a maximum $r r n$ occupancy of $\sim 72$ RNAPs (using the average $r r n$ occupancy at the maximal $E$. coli growth rate of $24 \mathrm{~min}$ ), the operons operate at only $\sim 32 \%$ of their full capacity, hence being far from saturation and having significant spare capacity.

Regarding $\Delta 5$ (T 57 min), we expect to have $\sim 240$ RNAPs engaged in $r r n$ transcription and $\sim 5 \mathrm{rrn}$ operons per cell, leading to an estimate of $\sim 50$ RNAPs per operon, and $\sim 70 \%$ of max occupancy; even if we use our experimental result of $\sim 3.2 \mathrm{rrn}$ foci/cell (see last Results section), and assume conservatively that a focus contains only one rrn operon, we recover an upper bound that does not exceed the $r r n$ operon capacity. These estimates strongly suggest that the remaining $r r n$ genes in $\Delta 5$ can accommodate the number of RNAPs required for the observed growth rate. Similarly, even for $\Delta 6$, the doubling time of 73 min can be maintained by an $\sim 85 \%$ occupancy of the remaining $\sim 2 \mathrm{rrnE}$ copies per cell. In essence, the $r r n$ transcription requirements for the growth rates of the deletion strains in M9Glu can be fulfilled by relocating RNAPs to the remaining $r r n$ operons. Regarding $\Delta 7$, which has a growth rate similar to $\mathrm{WT}$, the presence of $\sim 10$ copies of the rrn-containing plasmid (pK4-16, based on pSC101, see also Ref. (28)) also provides enough rrn copies to sustain the required levels of rRNA.

A more complex picture emerges for the $\Delta r r n$ strains in rich media. In RDM, the WT (T 36 min) has $\sim 22 \mathrm{rrn}$ operons per cell and $\sim 800$ rRNAPs, with an average of $\sim 37$ RNAPs/rrn, which corresponds to operating at $\sim 50 \%$ full capacity. As in the M9Glu case, since $\Delta 7$ has many copies $(\sim 15)$ of the $r r n B-$ containing plasmid, it can maintain high $r r n$ transcription levels despite the loss of all chromosomal $r r n$; indeed, $\Delta 7$ shows the smallest fitness cost (increase in doubling time) amongst the $\Delta r r n$ strains.

However, in the case of $\Delta 5$ (T 46 min, $\sim 420$ RNAPs), we expect $\sim 5.6 \mathrm{rrn}$ per cell based on the measured growth rate, hence $\sim 75$ RNAPs/rrn, which exceeds the maximum capacity by $\sim 5 \%$; this means that $\Delta 5$ is at the limit of being able to sustain growth by fully loading all remaining $r r n$ operons with RNAPs. This limit is substantially exceeded in $\triangle 6$, the slowest growing strain in RDM (T 51 min), which requires $\sim 320$ rRNAPs to maintain its growth rate, corresponding to $\sim 130$ RNAPs/rrn, which is physically impossible.

This result strongly suggests that mechanisms other than simple RNAP relocation to the number of $r r n$ copies expected purely on the basis of growth rate are needed to explain the ability of $\Delta 6$ (and, possibly, of $\Delta 5$ ) to sustain the observed growth rate in RDM. 


\section{$r r n$ transcription in rich media for $\Delta r r n$ is further compensated by increased gene dosage}

Our estimates of RNAP occupancy of $r r n$ in RDM clearly showed that, at least for the $\Delta 6$ strain, the cells cannot sustain the measured growth rate purely on the basis of the cellular number of $r$ operons expected for the measured growth rate. This led us to hypothesise that more $r r n$ are generated, likely due to increased replication initiation frequency. To explore this hypothesis, we used imaging to measure the number of $r r n E$ copies in WT and in $\triangle 6$ growning in RDM; the $r r n E$ copies were visualised as fluorescent foci (due to the binding of fluorescent ParB molecules) forming on parS sequences inserted in chromosomal positions adjacent to the rrnE operons in WT and $\Delta 6$ (see Methods).

Fluorescence imaging of the WT strain, followed by an automated analysis of foci (see Methods), showed that most cells have 1-4 bright foci (Fig. 5A), with the number increasing with cell length (Fig. $\mathrm{S} 13 \mathrm{E}$ ), and an average of $\sim 2.8$ foci per cell (SD 1.24; Fig. $5 \mathrm{~B}$ ), with the distribution width reflecting the fact that cells throughout the cell cycle are included in the analysis. The average number of foci is in very good agreement with the $\sim 3.3 \mathrm{rrnE}$ copies in RDM expected on the basis of growth rate (T 36 $\min )$.

Similar analysis of the $\Delta 6$ strain showed that most cells carry a higher number of bright foci (up to 8 foci), for an average of $\sim 4.3$ foci per cell (SD 2.5; Fig. 5C-D). This increase leads to a number of $r r n E$ copies that is $\sim 70 \%$ higher than what is expected purely on the basis of growth rate ( 2.5 copies), and leads to an estimated $\sim 74$ rRNAPs per operon, matching the maximum loading capacity of a $r r n$ operon. We performed a similar comparison between WT and $\Delta 5$ (Fig. S13A-D); considering the growth rates of the two strains, we were expecting $\sim 28 \%$ more $r r n C$ foci in WT compared to $\Delta 5$. However, the average of $r r n C$ foci in WT is similar to that of $\Delta 5$ (4.8 foci in WT vs 4.9 in $\Delta 5$ ), consistent with the presence of additional replication initiation to increase gene dosage in the case of $\Delta 5$, albeit the effect is not as pronounced as in $\Delta 6$.

\section{Two-colour imaging reveals RNAP clusters correspond to $r$ rn foci}

To provide direct evidence for the link between RNAP clusters and $r r n$ operons in the $\Delta r r n$ strains, we performed two-colour colocalization assays by combining fluorescence in situ hybridization (FISH) imaging of $r r n$ foci with single-molecule RNAP localisation in $\Delta 5$. Similarly to published work (13), we used a fluorescent FISH probe that targeted the 5 ' leader region of the $16 \mathrm{~S}$ precursor rRNA (pre-rRNA), which is absent from mature rRNA and ribosomes (see Methods). Signal from our FISH probe in fixed cells in M9Glu allows us to capture transcribing $r r n$ foci, visualized as bright diffraction-limited spots in pole-proximal regions (Fig. 6A). When used in conjunction with PALM data, the relative colocalization identified active $r r n$ clusters in $\Delta 5$ and estimated their copy numbers in M9Glu and RDM media to be $\sim 3.2 \pm 0.1$ and $\sim 6.8 \pm 0.2$, respectively (Fig. $6 \mathrm{D}$ ). To record the position of $r r n$ foci, its centroid was determined by a Gaussian fitting and superimposed on RNAP localizations and RNAP clusters; we observed that most RNAP clusters (especially large clusters containing $>50$ localisations; $C>50$ ) locate within $200 \mathrm{~nm}$ from the $\mathrm{rrn}$ centroid (Fig. 6A), suggesting significant co-localization between RNAP clusters and $r r n$ foci.

To quantify the degree of colocalization, we performed pair-correlation analysis between the $r$ loci and RNAP clusters (either using all clusters with $\mathrm{N}>=4$, or large $\mathrm{C}>50$ clusters). Our results show a high 
correlation between $r$ rn foci and RNAP clusters, supporting their colocalization. Specifically, we found that $\sim 46 \%$ of all RNAP clusters, and $\sim 77 \%$ of RNAP large clusters localize within $200 \mathrm{~nm}$ of $r \mathrm{rn}$ foci, compared with $\sim 23 \%$ expected on the basis of simulated random RNAP localisations (Fig. 6B), which employs similar analysis algorithms as our pair-correlation analysis in Fig. 4.

To visualize the distribution of RNAP clusters, we generated the heatmap of RNAP clusters from the normalized positions of clustered RNAPs in $\triangle 5$ grown in M9Glu (Fig. $6 \mathrm{C}$ ). Our results clearly show that RNAP clusters are concentrated at pole-proximal regions, a fact also reflected in projections along the cell-length axis. The projection of the normalized positions of $r r n$ foci also display pole-proximal peaks, which highly overlap with the peaks of RNAP clusters (Fig. 6C). These results clearly establish the physical proximity of the RNAP clusters and $r r n$ foci, and further support the suggestion that RNAPs relocate to the remaining $r r n$ operons to sustain high levels of $r r n$ transcription and largely maintain the growth rate achieved in the absence of any rrn deletions.

\section{DISCUSSION}

The spatial organization of RNAP in bacteria has been a long-standing question ever since the first observations of transcription foci in cells grown in rich media (1-3,8,47-49), and the linkage between transcription foci and rRNA synthesis has remained controversial (1). Here, we applied super-resolution imaging and single-molecule tracking on strains with a heavily reduced number of $r r n$ operons to elucidate the relation between RNAP spatial organization and rRNA synthesis, and study how cells redeploy their transcription machinery to sustain a healthy growth rate with only 1 or 2 chromosomal $r r n$ operons. Notably, most bacterial species ( $80 \%)$ have $1-4$ rrn copies in their genome, with $\sim 35 \%$ having just 1-2 copies (50); a large number of $r$ copies enables the provision of high numbers of ribosomes per cell, which in turn allows bacteria harbouring a large rrn number to adapt faster to nutritional upshifts and switch to fast growth (and in general, respond more rapidly to changes in the nutrient availability) (50).

RNAPs maintain their chromosome engagement in $\Delta r r n$ strains by increasing the loading of the remaining $r$ rn operons. Our RNAP mobility analysis showed that the fraction of immobile RNAPs, a proxy for the fraction of RNAPs engaged in transcription (plus any RNAPs involved in condensates), is surprisingly robust to the loss of 5 and 6 chromosomal $r r n$ copies, as well as to the loss of all chromosomal $r$ copies when cells are supplemented by a low-copy-number plasmid harbouring a single rrn operon. In M9Glu, a medium that supports a doubling time of $47 \mathrm{~min}$ in WT, about half of all RNAPs were immobile both for WT (as seen in Refs $(12,51)$ ) and all $\Delta r r n$ strains. Even in rich media (RDM; supporting a doubling time of $36 \mathrm{~min}$ in WT), heavy loss of $r r n$ operons led to only a modest decrease in the immobile RNAP fraction ( $63 \%$ for WT; $57-58 \%$ for the $\Delta r r n$ strains).

The robustness of the RNAP immobile fraction to the loss of most chromosomal rrn genes raises the question of how RNAPs "released" from the deleted $r r n$ operons redistribute to other immobile fractions, and how this redistribution minimises any growth-rate defects in $\Delta r r n$ strains. Under our growth conditions in the WT strain (Fig. 7, top), we expect that rrn promoters are not saturated with RNAP; as a result, an increase in the concentration of available free RNA polymerase should lead to increased 
$r r n$ promoter activities (46). Our results support a scenario where the remaining $r r n$ copies in the cell are more heavily loaded by RNAPs (Fig. 7, middle).

Such RNAP re-distribution had been observed in a study of a more limited $r$ n deletion in rich medium LB; specifically, deletion of $4 \mathrm{rrn}$ operons $(\Delta 4)$ resulted in the remaining $r r n$ operons accommodating 71 RNAPs, an increase from 53 RNAPs/rrn in the strain with all operons intact, with the increased occupancy being linked to increased transcription initiation and elongation (38). Notably, the growth slow-down seen in $\Delta 4$ ( $24 \mathrm{~min}$ for WT vs $\sim 30 \mathrm{~min}$ for $\Delta 4)$ is similar to that we see for $\Delta 5$ in RDM.

Our observations are consistent with the "saturation model" for the passive regulation on gene expression $(46,52)$, and with studies showing that a 2- to 3-fold overexpression of the RNAP- $\sigma 70$ holoenzyme leads to a 2-fold increase of $r n B P 1$ transcription, and a large increase in the tendency to transcribe rrn genes versus mRNA genes (53). In general, our results clearly show that the rrn promoters are competing very effectively against mRNA promoters.

RNAP clusters do form on rrn operons. Our results clearly establish that RNAPs are more clustered in the $\Delta r r n$ strains, and that RNAP clusters are in physical proximity to the $r r n$ foci, adding further support to the proposal that RNAPs relocate to the remaining $r r n$ operons to sustain high levels of $r r n$ transcription and largely maintain the growth rate achieved in the absence of any rrn deletions. Our results are consistent with results from Weng et al (13), where it was shown that large RNAP clusters are maintained in a strain with a single $r r n$ on the chromosome in rich media, as well as during low levels of transcription (13).

The presence of clustering on $r r n$ (rRNAPs) does not exclude the presence of other forms of clustering, such as condensates (CRNAP) or heavy transcription on mRNA genes. It demonstrates, however, the ability of RNAP to redistribute and reprogram gene expression due to the cellular response to changes in the local environment or chromosome context $(1,13)$.

The location of remaining rrn operons dictates the location of the clusters in M9Glu. Considering the genomic map (Fig. 1B), the remaining $r r n$ in the deletion mutants are either near ori (38), which is situated at pole-proximal regions along the cell long-axis (44), or on plasmids known to localise preferentially at the polar endcaps; these positions are consistent with the new peak of localisations (along the long cell axis) that appears in all $3 \Delta r r n$ strains (Fig. 2D). Relocation of released immobile RNAPs in MGlu in $\Delta r r n$ to pole-proximal positions is thus dictated by the places of the remaining $r r n$ operons.

The presence of a well-defined location for the relocated rRNAPs makes it unlikely that the released rRNAPs relocate to transcribe mRNA or join condensates not associated with transcription, since such RNAP pools are expected to have a much less localized position on the heat maps. In essence, it is the clustered rRNAPs that recruit cRNAPs (at least in the M9Glu case), and not the other way around.

Heavy loss of $r$ rn operons in rich media is further compensated via increased gene dosage. Our estimates of rRNAPs per rrn copy indicated that, to maintain a moderate growth rate in rich media, mechanisms additional to RNAP relocation are necessary; our measurements of fluorescent foci 
corresponding to specific $r$ rn operons clearly demonstrated highly increased gene dosage, especially for the $\Delta 6$ strain. The increase in $r r n$ copy numbers per cell is likely to result from increased frequency of replication initiation, thus linking gene expression with DNA replication (Fig. 7, bottom).

Such an intriguing connection has also been observed in S. pneumoniae, E. coli and other bacteria, in cases where stress induced by antibiotic treatments that target DNA replication (and stall replication forks) activates bacterial competence via a similar increased gene dosage of competence-related genes, which have an evolutionary conserved location near the replication origin (54).

In the case of the increased $r r n$ gene dosage in $\Delta 5$ and $\Delta 6$ in rich media, we suggest that replication forks may be stalling due to co-directional collisions of the replication fork with RNAPs within rrn operons, since they will be fully occupied by elongating RNAPs and operate at maximum capacity; in contrast, replication initiation is unaffected, eventually increasing gene dosage for ori-proximal genes (Fig. 7 , bottom). Such co-directional replication-transcription conflicts on highly transcribed $r r n$ operons have indeed been reported (55), and depended on the presence of transcription and on rapid growth, during which $>100$ RNAPs per $r$ operon were estimated to be present in B. subtilis (55).

\section{ACKNOWLEDGMENTS}

The authors thank Dr. Olivier Espeli for providing the pFH2973 plasmid, the parSPMT1 genomic strain used for the parS strain construction and other molecular biology tools; CGSC stock center for providing the original $\Delta r r n$ strains; the Oxford Synthetic Biology Doctoral Training Centre for access to a BMG Labtech microplate reader; and the MICRON Advanced Bioimaging Facility (supported by Wellcome Strategic Awards 091911/B/10/Z and 107457/Z/15/Z) for access to a PALM microscope in their facilities.

\section{FUNDING}

A.N.K. was supported by Wellcome Trust grant 110164/Z/15/Z, European Council Grant 261227, and the UK Biotechnology and Biological Sciences Research Council grants BB/N018656/1 and BB/S008896/1. J.F. was supported by the National Natural Science Foundation of China grant (No.32101049), a Medicine-engineering interdisciplinary grant by UESTC (ZYGX2021YGLH006) and a Talent recruitment program by UESTC. M.S. was supported by Wellcome Trust grants 204684/Z/16/Z and $224212 / Z / 21 / Z$.

\section{AUTHOR CONTRIBUTIONS}

A.N.K. and M.S. conceived the project. A.N.K., J.F, and H.e.S. designed the study. J.F., M.S., H.e.S., O.P., and J.K. performed experiments and analysed data. O.P. performed simulations, provided software, and analysed data. A.N.K. analysed data. J.F. and A.N.K. wrote the paper, and all authors had the chance to read and edit the paper.

\section{DATA AVAILABILITY}

Movies and images of cells as well as localisation files for single molecules will be available upon request. 


\section{FIGURES}
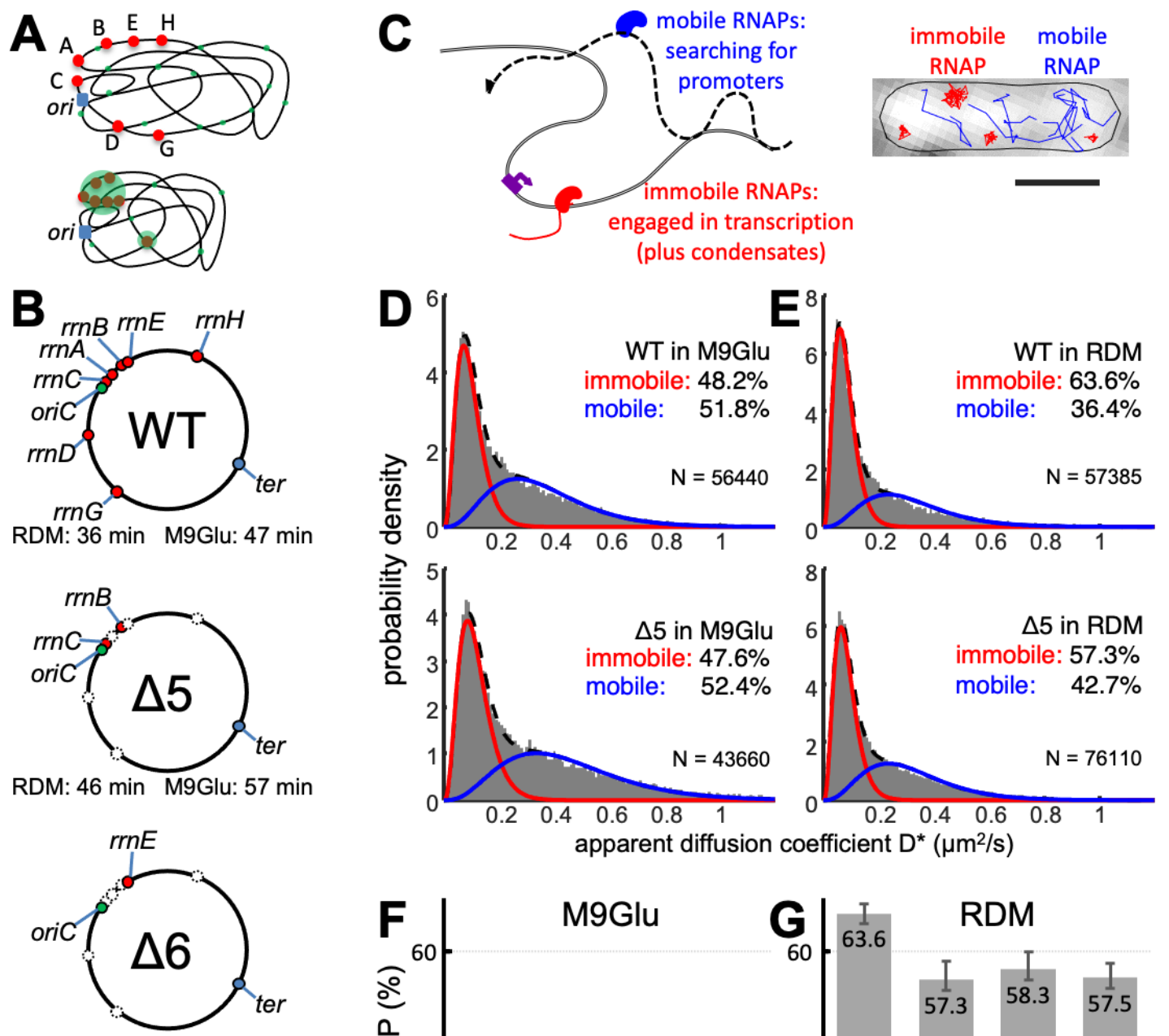

RDM: 51 min M9Glu: 73 min
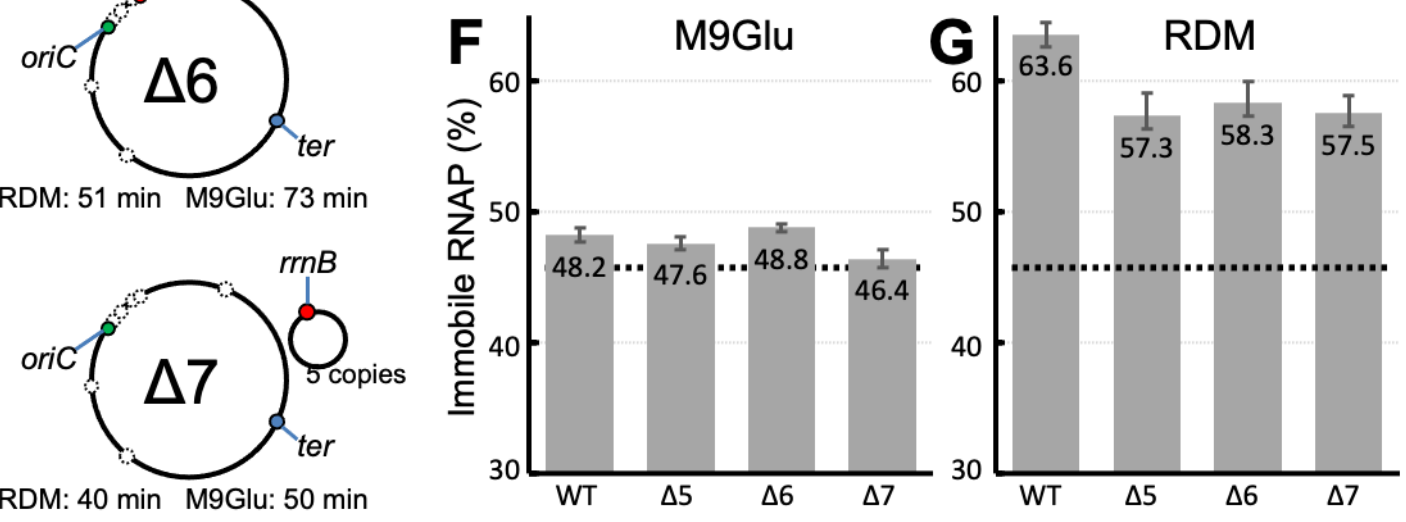

Fig. 1. Measuring chromosome engagement of RNA polymerase at single-molecule level in $E$. coli strains with deletions of most chromosomal rrn operons.

A. Models of RNAP distribution in cells based on observations of transcription foci and clustered RNAPs. Ribosomal operons are shown in red; RNAPs engaged in transcription are shown in green; origin of replication is shown in blue. Top: transcription-engaged RNAPs in minimal media are distributed throughout the nucleoid, with a bias towards the nucleoid periphery. Bottom: in rich media, transcription is concentrated on rrn operons, most of which appear in close proximity, forming large RNAP clusters.

B. WT and $r r n$ deletion strains used in this study; $r r n$ loci are marked in the genomic map or in the supplemented plasmid. Doubling times of each strain in RDM and M9Glu media are also shown.

C. Main mobility species of RNAP molecules and their characterisation using single-molecule tracking PALM. Scale bar, $1 \mu \mathrm{m}$.

D-E. Histograms of apparent diffusion coefficient $\left(D^{*}\right)$ fitted with two-gamma distributions for WT and $\triangle 5$ in the M9Glu (D) and RDM media (E), along with the fractions of immobile and mobile RNAPs. N denotes the number of tracks per histogram.

F-G. Fractions of immobile RNAP fractions for all strains in M9Glu (F) and RDM media (G). Dashed lines, median immobile RNAP fraction in M9Glu; error bars, S.E.M. from three individual measurements. 

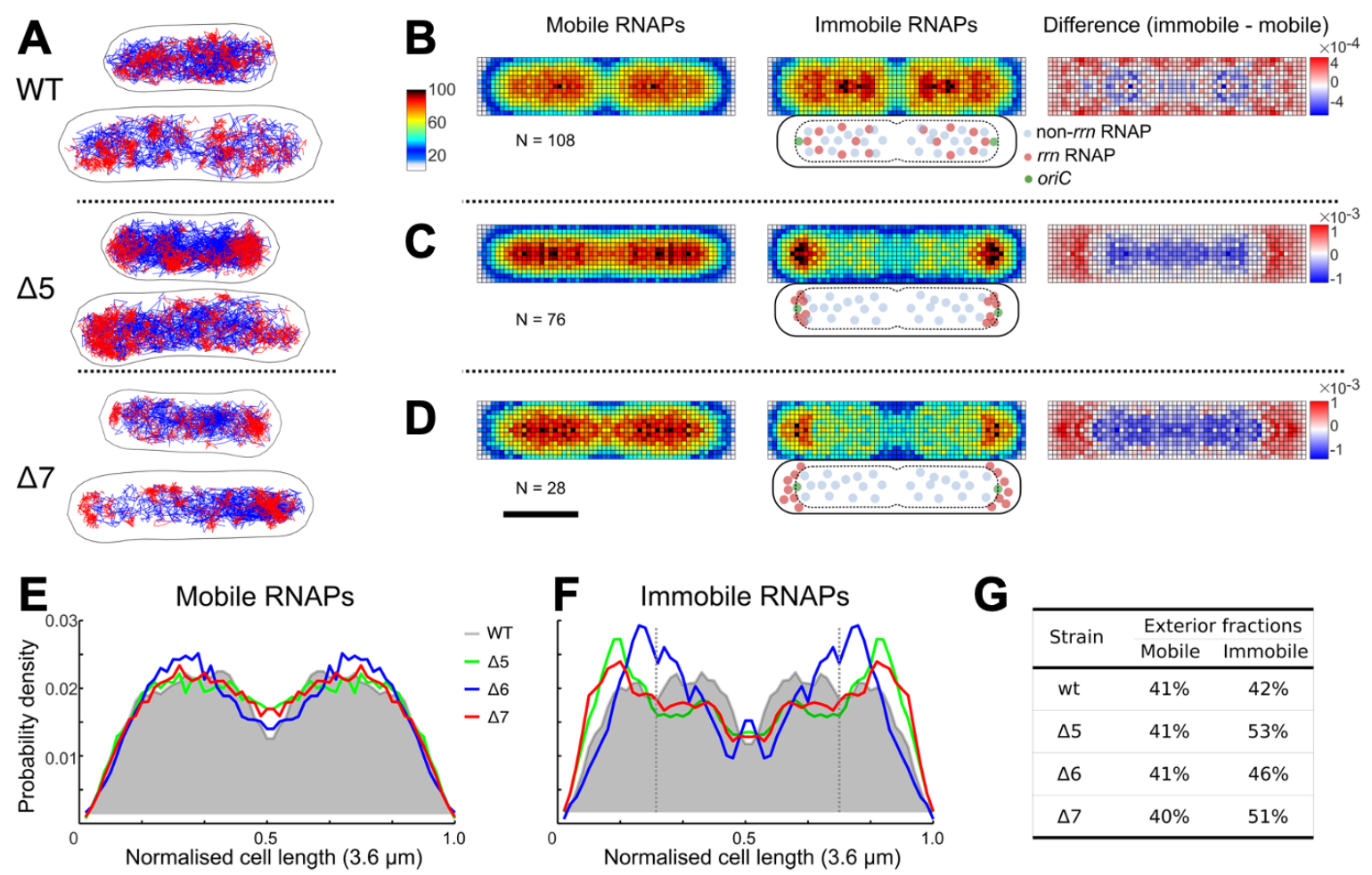

F Immobile RNAPs

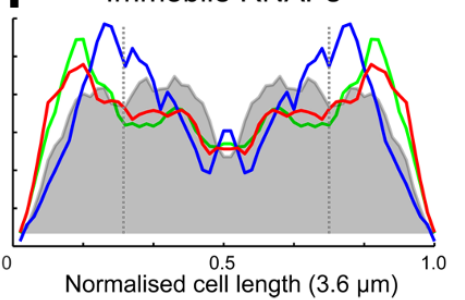

G

\begin{tabular}{ccc}
\hline Strain & \multicolumn{2}{c}{ Exterior } \\
& Mobile & Immobtions \\
\hline $\mathrm{wt}$ & $41 \%$ & $42 \%$ \\
\hline$\Delta 5$ & $41 \%$ & $53 \%$ \\
\hline$\Delta 6$ & $41 \%$ & $46 \%$ \\
$\Delta 7$ & $40 \%$ & $51 \%$ \\
\hline
\end{tabular}

Fig. 2. Immobile RNAPs in the $\Delta r r n$ strains redistribute to polar-proximal regions in M9Glu media.

A. Tracks of immobile (red) and mobile (blue) RNAP molecules for representative cell examples. The $D^{*}$ threshold for the track color-coding was $0.16 \mu \mathrm{m}^{2} / \mathrm{s}$. Scale bar (shown in panel D), $1 \mu \mathrm{m}$.

B-D. Left and middle: heatmaps from multiple cells within $3.2-4.0 \mu \mathrm{m}$ range of cell lengths for both mobile and immobile RNAPs. Right, difference map calculated by subtraction of mobile RNAPs from immobile RNAPs. Cartoons below the immobile RNAP heatmaps display the relative positions of $r r n-$ related and non-rrn-related RNAPs relative to cell membrane, nucleoid and the ori region. Scale bar, 1 $\mu \mathrm{m}$.

E-F. Projections of mobile (E) and immobile (F) RNAP localisations along the long-axis of the heatmaps. The projection from WT is shaded grey. Dashed vertical lines indicate the $25 \%$ position along the long axis.

G. The fractions of RNAPs localised in the exterior $25 \%$ region along the long axis (i.e., from 0 to 0.25 normalised cell length in Fig. 2F). 

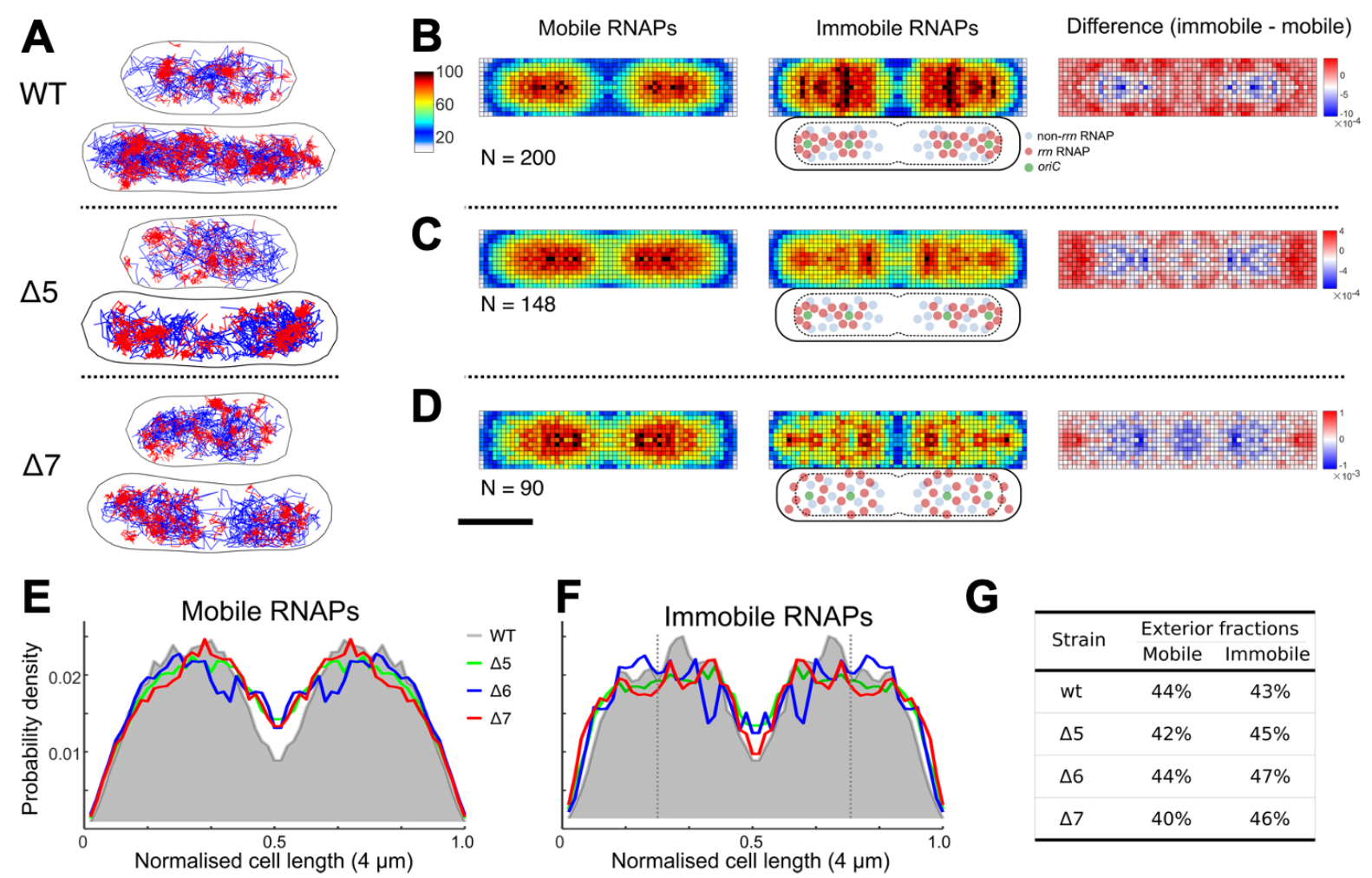

Fig. 3. RNAP spatial distribution in the WT and $\Delta r r n$ strains in rich media (RDM).

A. Tracks of immobile (red) and mobile (blue) RNAP molecules for representative cell examples. The $D^{*}$ threshold for the track color-coding was $0.16 \mu \mathrm{m}^{2} / \mathrm{s}$. Scale bar (shown in panel D), $1 \mu \mathrm{m}$.

B-D. Left and middle: heatmaps from multiple cells within 3.5-4.5 $\mu \mathrm{m}$ range of cell lengths for both mobile and immobile RNAPs. Right, difference map calculated by subtraction of mobile RNAPs from immobile RNAPs. Cartoons below the immobile RNAP heatmaps display the positions of $r r n$-related and non-rrn-related RNAPs relative to cell membrane, nucleoid and the ori region. Scale bar, $1 \mu \mathrm{m}$.

E-F. Projections of mobile (E) and immobile (F) RNAP localisations along the long-axis of the heatmaps. The projection from WT is shaded grey. Dashed vertical lines indicate the $25 \%$ position along the long axis.

G. The fractions of RNAPs localised in the exterior $25 \%$ region along the long axis (i.e., from 0 to 0.25 normalised cell length in Fig. 3F). 

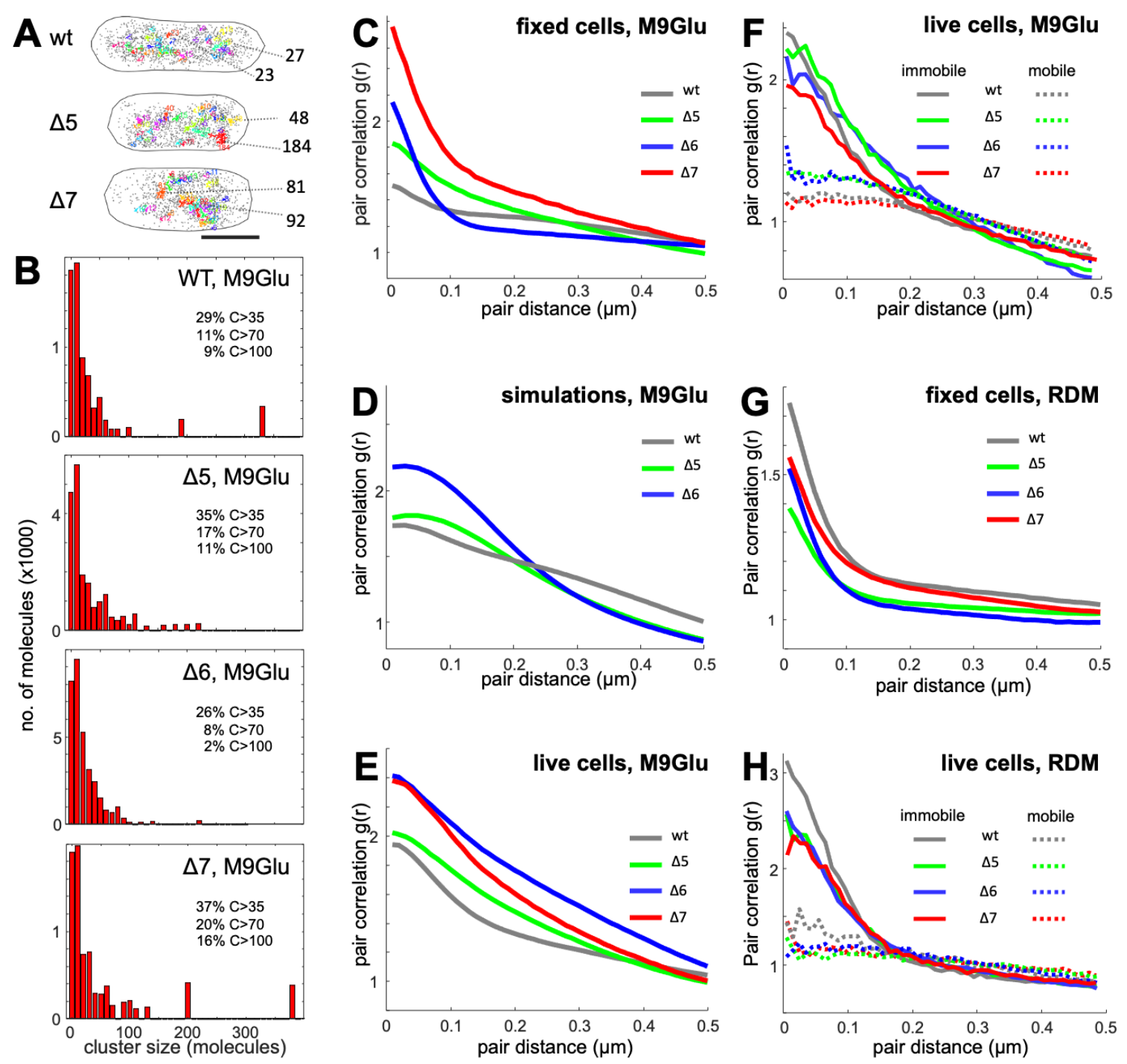

Fig. 4. RNAP clustering and pair-correlation analysis of RNAP localisations in M9Glu and rich media.

A. Representative examples of RNAP clusters in single fixed cells for WT and two $\Delta r r n$ strains. RNAP clusters are displayed in different colors; non-clustered localizations are displayed as isolated grey points. Scale bar, $1 \mu \mathrm{m}$. Two clusters from each cell along with the cluster size (number of molecules) are shown as examples.

B. Histograms of cluster size of RNAPs in fixed cells in M9Glu media. The fractions of large clusters of different size are listed alongside.

C-H. Pair-correlation analysis of RNAP localisations in fixed and live cells of WT and $\Delta r r n$ strains in M9Glu and RDM. C. Analysis in fixed cells of all strains in M9Glu. D. Analysis of simulated RNAP localisations for WT and two $\Delta r r n$ strains in M9Glu. E. Analysis in live cells for all strains in M9Glu. F. Analysis of immobile and mobile RNAP tracks in live cells for all strains in M9Glu. G. Analysis of RNAP localisations in fixed cells for all strains in RDM. H. Analysis of immobile and mobile RNAP tracks in live cells for all strains in RDM. 

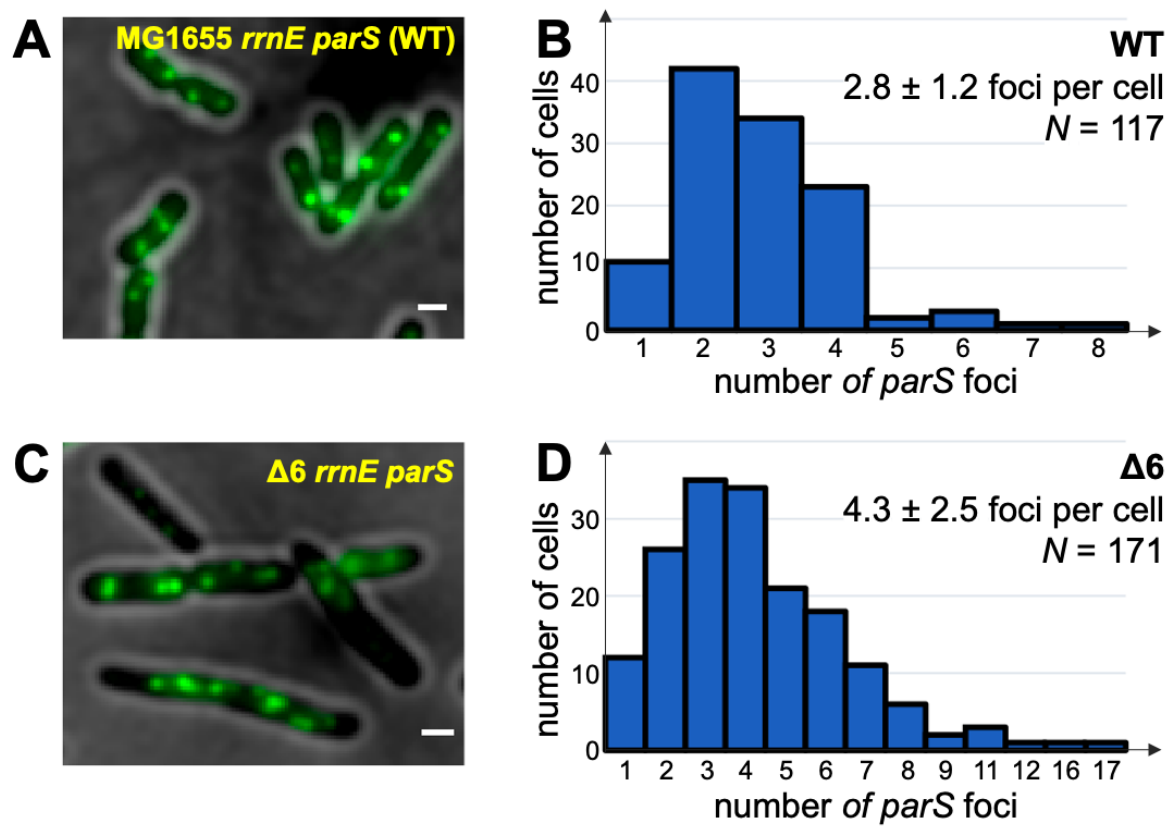

Fig. 5. Imaging of rrnE operons shows increased gene dosage in RDM for $\Delta 6$ compared with WT. Fluorescence imaging of $r$ rnE foci in the rpoC:PAmCherry rrnE parS strain harbouring plasmid pFH2973 expressing parB-yGFP. Cells were grown in RDM until $\mathrm{OD}_{600} \sim 0.2$, immobilized on agarose pads, and imaged upon 473-nm laser excitation and using 500-ms exposures.

A. Example of an image of cells containing parB foci at $r$ rnE operons (appearing as bright green foci). Scale bar, $1 \mu \mathrm{m}$.

B. Frequency distribution of the number of foci per cell for the WT strain, along with the mean and standard deviation of the distribution. Cells were segmented and foci were localised and counted using microbeJ (see Methods). $N$ denotes number of cells.

C-D. As in A and B, but for the $\Delta 6$ strain. The mean number of $r r n E$ foci in $\Delta 6$ increases by $\sim 70 \%$ compared to WT. 

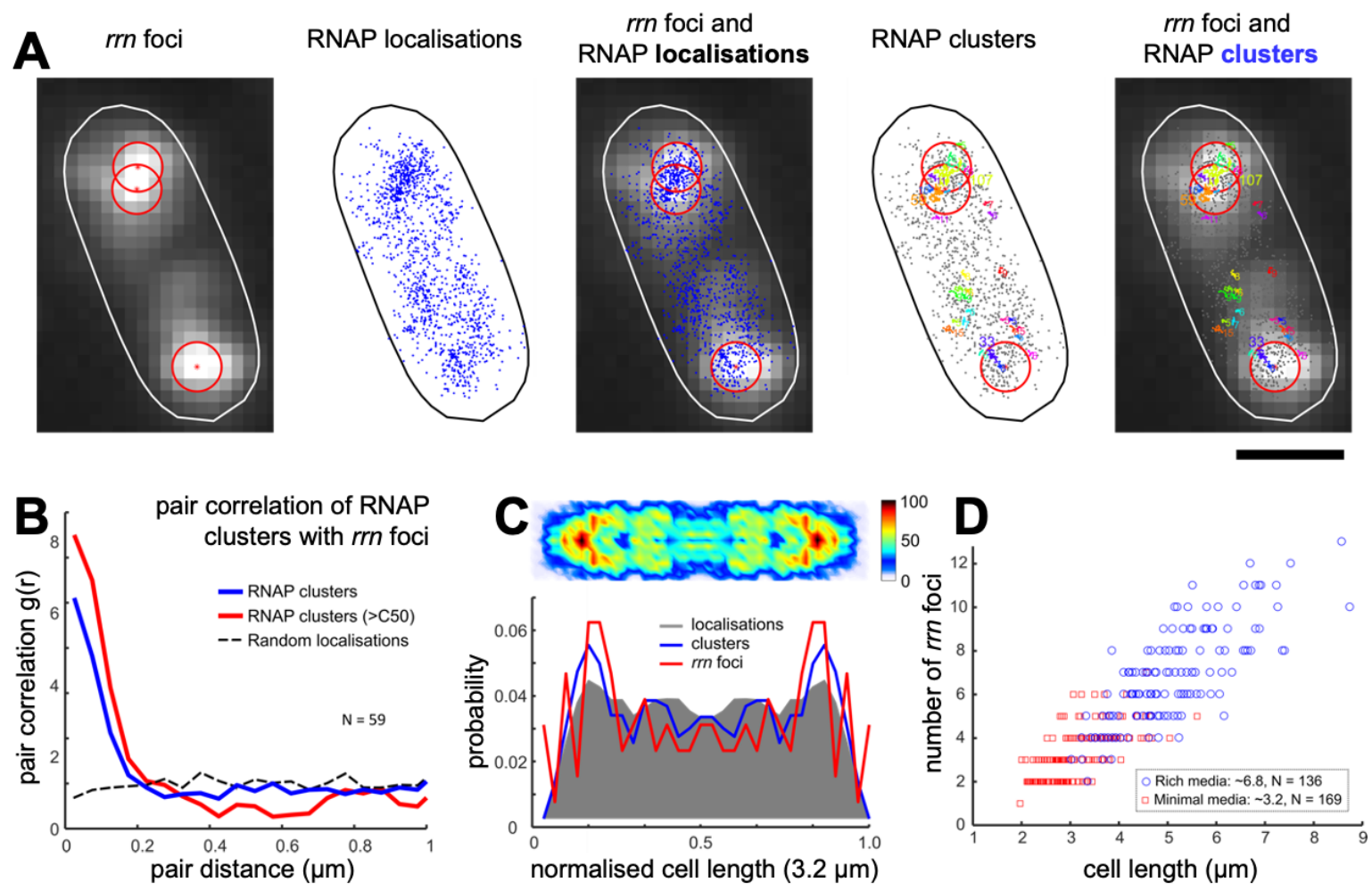

Fig. 6. Co-localization of $r \boldsymbol{r n}$ foci with RNAP clusters in fixed cells.

A. Representative example of a fixed cell showing FISH-labelled $r r n$ foci in $\Delta 5$ along with RNAP localisations and clusters. Each centroid corresponds to one $r r n$ focus obtained by Gaussian fitting and marked by a red asterisk and a $200 \mathrm{~nm}$-radius red circle. RNAP localisations in fixed cells were analysed using DBSCAN to identify clusters; different clusters, along with their number of RNAPs, are shown in different colors. Scale bar, $1 \mu \mathrm{m}$.

B. Pair-correlation analysis of $r r n$ foci with all RNAP clusters, as well as large RNAP clusters $(C>50)$ according to the pairwise distance show high co-localization between $r r n$ foci with RNAP clusters and C>50 clusters: $\sim 46 \%$ of RNAP clusters and $\sim 77 \%$ of RNAP large clusters locate within a $200-\mathrm{nm}$ radius of $r r n$ foci (blue line), compared with $\sim 23 \%$ obtained for simulated localisations randomly distributed throughout the nucleoid area (dashed black line).

C. Heatmap of RNAP clusters and their projections (blue line). Projections of localisations and $r r n$ foci are displayed in grey shaded area and red line, respectively.

D. Scatter plot of $r r n$ foci number in $\Delta 5$ in M9Glu and RDM as a function of cell length. Mean number of rrn foci is $3.2 \pm 0.1$ (SEM) in M9Glu, and $6.8 \pm 0.2$ (SEM) in RDM. 
WT
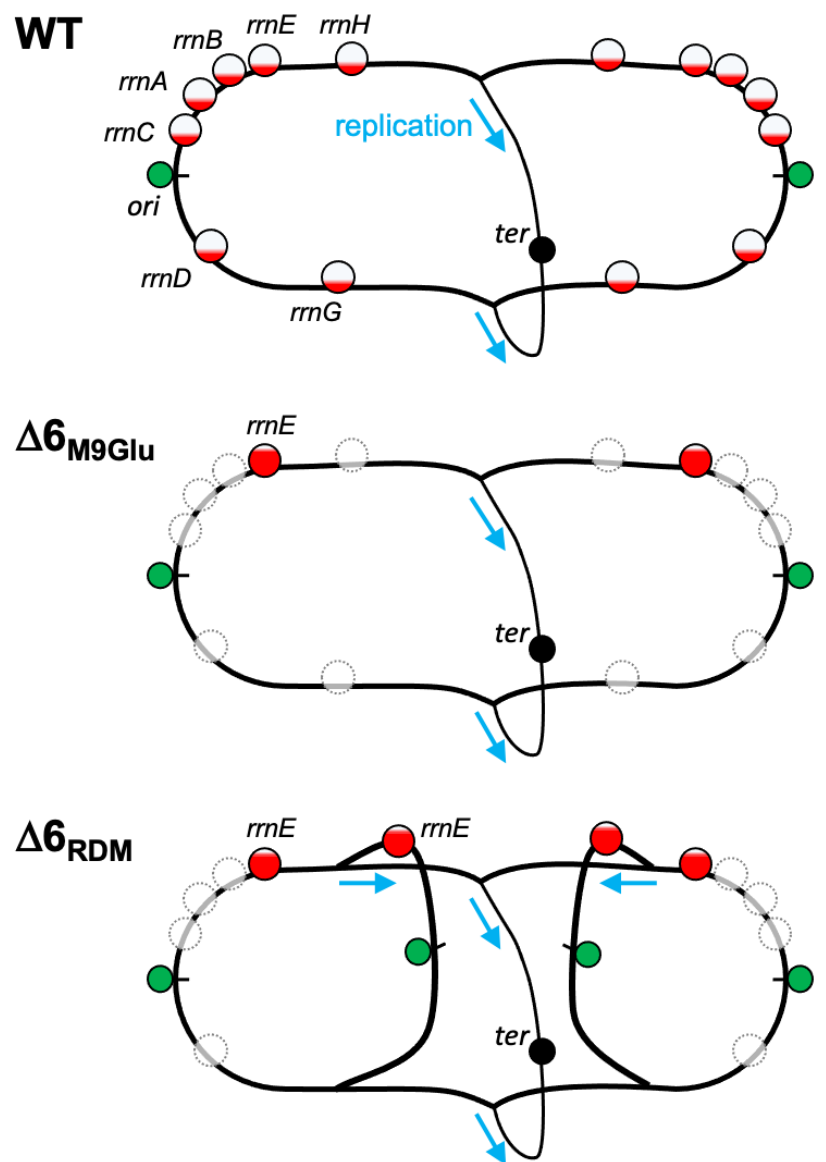

M9Glu (moderate growth)

○ $\sim 30 \%$ rrn occupancy

rich media (fast growth)

$\ominus \sim 50 \%$ rrn occupancy

M9Glu (moderate growth)
$>80 \%$ rrnE occupancy

rich media (fast growth)

O $>80 \%$ rrnE occupancy

and

increased $r$ rnE gene dosage

Fig. 7. RNAP redistribution and increased gene dosage cooperate to maximise $r$ rn transcription and minimize growth defects in $E$. coli strains with a small number of $r r n$ operons. The $E$. coli strain with all 7 chromosomal $r r n$ copies carries a large number of $r r n$ copies ( $>15$ copies) per cell either in M9Glu or rich media, with the operons being far from saturation (30-50\% occupancy) in both media, and RNAP clusters forming on $r r n$ spreading throughout the entire nucleoid. In contrast, in rrn deletion mutants lacking most $r r n$ copies from the chromosome (e.g., $\Delta 6$ ), the remaining $r r n$ copies are much more occupied by RNAPs ( $>80 \%$ in $\Delta 6$ in M9Glu) and RNAP clusters are more concentrated in the pole-proximal positions. More demanding growth conditions with regards to rRNA resources, such as in rich media, are facilitated by relative increase in replication initiation and a corresponding increase in the gene dosage for $r n$ (a $\sim 70 \%$ increased dosage for $\Delta 6)$, with RNAPs clusters spreading throughout the entire nucleoid. 
Table 1. Estimated occupancy of $r r n$ operons in WT and $\Delta r r n$ strains as a function of growth rate.

\begin{tabular}{|c|c|c|c|c|c|c|}
\hline Strain & Media & $\begin{array}{c}\text { Doubling } \\
\text { time }(\mathrm{min})\end{array}$ & $\begin{array}{c}\mathrm{N}_{\text {rRNAP }} \\
\text { per cell }\end{array}$ & $\begin{array}{c}r r n \text { copies } \\
\text { per cell }\end{array}$ & $\begin{array}{c}\mathrm{N}_{\text {rRNAP }} \\
\text { per } r n\end{array}$ & $\begin{array}{c}\text { RNAP occupancy } \\
\text { of each } r n^{* *}\end{array}$ \\
\hline WT & M9Glu & 47 & 397 & 17.5 & 23 & $32 \%$ \\
\hline$\Delta 5$ & M9Glu & 57 & 238 & 4.8 & 50 & $69 \%$ \\
\hline$\Delta 6$ & M9Glu & 73 & 122 & 2.0 & 61 & $85 \%$ \\
\hline WT & RDM & 36 & 805 & 22.0 & 37 & $51 \%$ \\
\hline$\Delta 5$ & RDM & 46 & 420 & 5.6 & 75 & $104 \%$ \\
\hline$\Delta 6$ & RDM & 51 & 320 & 2.5 & 128 & $178 \%$ \\
\hline
\end{tabular}

For the methods used to obtain the estimates, see Methods.

*Number of RNAP molecules transcribing $r r n$ operons in the different strains.

** $100 \%$ occupancy is set to 72 rRNAPs/rrn. 
bioRxiv preprint doi: https://doi.org/10.1101/2022.02.10.479894; this version posted February $11,2022$. The copyright holder for this

preprint (which was not certified by peer review) is the author/funder, who has granted bioRxiv a license to display the preprint in perpetuity. It is made available under aCC-BY 4.0 International license.

\section{REFERENCES}

1. Cabrera, J.E. and Jin, D.J. (2003) The distribution of RNA polymerase in Escherichia coli is dynamic and sensitive to environmental cues. Molecular Microbiology, 50, 1493-1505.

2. $\quad$ Reeder, R.H. (1990) rRNA synthesis in the nucleolus. Trends in Genetics, 6, 390-394.

3. Cook Peter, R. (1999) The Organization of Replication and Transcription. Science, 284, 17901795.

4. Cisse Ibrahim, I., Izeddin, I., Causse Sebastien, Z., Boudarene, L., Senecal, A., Muresan, L., Dugast-Darzacq, C., Hajj, B., Dahan, M. and Darzacq, X. (2013) Real-Time Dynamics of RNA Polymerase II Clustering in Live Human Cells. Science, 341, 664-667.

5. Cho, W.-K., Jayanth, N., English, B.P., Inoue, T., Andrews, J.O., Conway, W., Grimm, J.B., Spille, J.-H., Lavis, L.D., Lionnet, T. et al. (2016) RNA Polymerase II cluster dynamics predict mRNA output in living cells. eLife, 5, e13617.

6. Lyle John, M., Bullitt, E., Bienz, K. and Kirkegaard, K. (2002) Visualization and Functional Analysis of RNA-Dependent RNA Polymerase Lattices. Science, 296, 2218-2222.

7. Lewis, P.J., Thaker, S.D. and Errington, J. (2000) Compartmentalization of transcription and translation in Bacillus subtilis. The EMBO Journal, 19, 710-718.

8. Cook, P.R. (2010) A Model for all Genomes: The Role of Transcription Factories. Journal of Molecular Biology, 395, 1-10.

9. Endesfelder, U., Finan, K., Holden, Seamus J., Cook, Peter R., Kapanidis, Achillefs N. and Heilemann, M. (2013) Multiscale Spatial Organization of RNA Polymerase in Escherichia coli. Biophysical Journal, 105, 172-181.

10. Bakshi, S., Siryaporn, A., Goulian, M. and Weisshaar, J.C. (2012) Superresolution imaging of ribosomes and RNA polymerase in live Escherichia coli cells. Molecular Microbiology, 85, 2138.

11. Bakshi, S., Choi, H. and Weisshaar, J.C. (2015) The spatial biology of transcription and translation in rapidly growing Escherichia coli. Frontiers in Microbiology, 6, 636.

12. Stracy, M., Lesterlin, C., Garza de Leon, F., Uphoff, S., Zawadzki, P. and Kapanidis, A.N. (2015) Live-cell superresolution microscopy reveals the organization of RNA polymerase in the bacterial nucleoid. Proceedings of the National Academy of Sciences, 112, E4390-E4399.

13. Weng, X., Bohrer, C.H., Bettridge, K., Lagda, A.C., Cagliero, C., Jin, D.J. and Xiao, J. (2019) Spatial organization of RNA polymerase and its relationship with transcription in Escherichia coli. Proceedings of the National Academy of Sciences, 116, 20115-20123.

14. Alberti, S., Gladfelter, A. and Mittag, T. (2019) Considerations and Challenges in Studying Liquid-Liquid Phase Separation and Biomolecular Condensates. Cell, 176, 419-434.

15. Sabari, B.R., Dall'Agnese, A., Boija, A., Klein, I.A., Coffey, E.L., Shrinivas, K., Abraham, B.J., Hannett, N.M., Zamudio, A.V., Manteiga, J.C. et al. (2018) Coactivator condensation at superenhancers links phase separation and gene control. Science, 361, eaar3958.

16. Cho, W.-K., Spille, J.-H., Hecht, M., Lee, C., Li, C., Grube, V. and Cisse, I.I. (2018) Mediator and RNA polymerase II clusters associate in transcription-dependent condensates. Science, 361, 412-415.

17. Chong, S., Dugast-Darzacq, C., Liu, Z., Dong, P., Dailey, G.M., Cattoglio, C., Heckert, A., Banala, S., Lavis, L., Darzacq, X. et al. (2018) Imaging dynamic and selective low-complexity domain interactions that control gene transcription. Science, 361, eaar2555.

18. Al-Husini, N., Tomares, D.T., Bitar, O., Childers, W.S. and Schrader, J.M. (2018) $\alpha-$ Proteobacterial RNA Degradosomes Assemble Liquid-Liquid Phase-Separated RNP Bodies. Molecular Cell, 71, 1027-1039.e1014.

19. Hondele, M., Sachdev, R., Heinrich, S., Wang, J., Vallotton, P., Fontoura, B.M.A. and Weis, K. (2019) DEAD-box ATPases are global regulators of phase-separated organelles. Nature, 573, 144-148.

20. Monterroso, B., Zorrilla, S., Sobrinos-Sanguino, M., Robles-Ramos, M.A., López-Álvarez, M., Margolin, W., Keating, C.D. and Rivas, G. (2019) Bacterial FtsZ protein forms phaseseparated condensates with its nucleoid-associated inhibitor SImA. EMBO reports, 20, e45946.

21. Guilhas, B., Walter, J.-C., Rech, J., David, G., Walliser, N.O., Palmeri, J., Mathieu-Demaziere, C., Parmeggiani, A., Bouet, J.-Y., Le Gall, A. et al. (2020) ATP-Driven Separation of Liquid Phase Condensates in Bacteria. Molecular Cell, 79, 293-303.e294.

22. Ladouceur, A.-M., Parmar, B.S., Biedzinski, S., Wall, J., Tope, S.G., Cohn, D., Kim, A., Soubry, N., Reyes-Lamothe, R. and Weber, S.C. (2020) Clusters of bacterial RNA polymerase are biomolecular condensates that assemble through liquid-liquid phase separation. Proceedings of the National Academy of Sciences, 117, 18540-18549.

23. Paul, B.J., Ross, W., Gaal, T. and Gourse, R.L. (2004) rRNA Transcription in Escherichia coli. Annual Review of Genetics, 38, 749-770. 
bioRxiv preprint doi: https://doi.org/10.1101/2022.02.10.479894; this version posted February $11,2022$. The copyright holder for this preprint (which was not certified by peer review) is the author/funder, who has granted bioRxiv a license to display the preprint in perpetuity. It is made available under aCC-BY 4.0 International license.

24. Jin, D.J., Mata Martin, C., Sun, Z., Cagliero, C. and Zhou, Y.N. (2017) Nucleolus-like compartmentalization of the transcription machinery in fast-growing bacterial cells. Critical Reviews in Biochemistry and Molecular Biology, 52, 96-106.

25. Gaal, T., Bratton, B.P., Sanchez-Vazquez, P., Sliwicki, A., Sliwicki, K., Vegel, A., Pannu, R. and Gourse, R.L. (2016) Colocalization of distant chromosomal loci in space in E. coli: a bacterial nucleolus. Genes \& Development, 30, 2272-2285.

26. Stracy, M. and Kapanidis, A.N. (2017) Single-molecule and super-resolution imaging of transcription in living bacteria. Methods, 120, 103-114.

27. Kapanidis, A.N., Uphoff, S. and Stracy, M. (2018) Understanding Protein Mobility in Bacteria by Tracking Single Molecules. Journal of Molecular Biology, 430, 4443-4455.

28. Quan, S., Skovgaard, O., McLaughlin, R.E., Buurman, E.T. and Squires, C.L. (2015) Markerless Escherichia coli rrn Deletion Strains for Genetic Determination of Ribosomal Binding Sites. G3 Genes|Genomes|Genetics, 5, 2555-2557.

29. Nielsen, H.J., Ottesen, J.R., Youngren, B., Austin, S.J. and Hansen, F.G. (2006) The Escherichia coli chromosome is organized with the left and right chromosome arms in separate cell halves. Molecular Microbiology, 62, 331-338.

30. Datsenko, K.A. and Wanner, B.L. (2000) One-step inactivation of chromosomal genes in \&lt;em\&gt;Escherichia coli\&lt;/em\&gt; K-12 using PCR products. Proceedings of the National Academy of Sciences, 97, 6640.

31. Cattoni, D.I., Fiche, J.-B., Valeri, A., Mignot, T. and Nöllmann, M. (2013) Super-Resolution Imaging of Bacteria in a Microfluidics Device. PLOS ONE, 8, e76268.

32. Ducret, A., Quardokus, E.M. and Brun, Y.V. (2016) MicrobeJ, a tool for high throughput bacterial cell detection and quantitative analysis. Nature Microbiology, 1, 16077.

33. Wolter, S., Löschberger, A., Holm, T., Aufmkolk, S., Dabauvalle, M.-C., van de Linde, S. and Sauer, M. (2012) rapidSTORM: accurate, fast open-source software for localization microscopy. Nature Methods, 9, 1040-1041.

34. Ester, M., Kriegel, H.-P., Sander, J. and Xu, X. (1996), Kdd, Vol. 96, pp. 226-231.

35. Gray, W.T., Govers, S.K., Xiang, Y., Parry, B.R., Campos, M., Kim, S. and Jacobs-Wagner, C. (2019) Nucleoid Size Scaling and Intracellular Organization of Translation across Bacteria. Cell, 177, 1632-1648.e1620.

36. Klumpp, S. and Hwa, T. (2008) Growth-rate-dependent partitioning of RNA polymerases in bacteria. Proceedings of the National Academy of Sciences, 105, 20245-20250.

37. Bremer, H. and Dennis Patrick, P. (2008) Modulation of Chemical Composition and Other Parameters of the Cell at Different Exponential Growth Rates. EcoSal Plus, 3.

38. Condon, C., French, S., Squires, C. and Squires, C.L. (1993) Depletion of functional ribosomal RNA operons in Escherichia coli causes increased expression of the remaining intact copies. The EMBO Journal, 12, 4305-4315.

39. Condon, C., Liveris, D., Squires, C., Schwartz, I. and Squires, C.L. (1995) rRNA operon multiplicity in Escherichia coli and the physiological implications of rn inactivation. Journal of Bacteriology, 177, 4152-4156.

40. Cabrera Julio, E., Cagliero, C., Quan, S., Squires Catherine, L. and Jin Ding, J. (2009) Active Transcription of rRNA Operons Condenses the Nucleoid in Escherichia coli: Examining the Effect of Transcription on Nucleoid Structure in the Absence of Transertion. Journal of Bacteriology, 191, 4180-4185.

41. Stracy, M., Schweizer, J., Sherratt, D.J., Kapanidis, A.N., Uphoff, S. and Lesterlin, C. (2021) Transient non-specific DNA binding dominates the target search of bacterial DNA-binding proteins. Molecular Cell, 81, 1499-1514.e1496.

42. Jin, D.J., Cagliero, C. and Zhou, Y.N. (2012) Growth rate regulation in Escherichia coli. FEMS Microbiology Reviews, 36, 269-287.

43. Jin, D.J., Cagliero, C. and Zhou, Y.N. (2013) Role of RNA Polymerase and Transcription in the Organization of the Bacterial Nucleoid. Chemical Reviews, 113, 8662-8682.

44. Sherratt David, J. (2003) Bacterial Chromosome Dynamics. Science, 301, 780-785.

45. Wang, J.D. and Levin, P.A. (2009) Metabolism, cell growth and the bacterial cell cycle. Nature Reviews Microbiology, 7, 822-827.

46. Dennis Patrick, P., Ehrenberg, M. and Bremer, H. (2004) Control of rRNA Synthesis in Escherichia coli: a Systems Biology Approach. Microbiology and Molecular Biology Reviews, 68, 639-668.

47. Cabrera Julio, E. and Jin Ding, J. (2006) Active Transcription of rRNA Operons Is a Driving Force for the Distribution of RNA Polymerase in Bacteria: Effect of Extrachromosomal Copies of rrnB on the In Vivo Localization of RNA Polymerase. Journal of Bacteriology, 188, 40074014. 
bioRxiv preprint doi: https://doi.org/10.1101/2022.02.10.479894; this version posted February $11,2022$. The copyright holder for this

preprint (which was not certified by peer review) is the author/funder, who has granted bioRxiv a license to display the preprint in perpetuity. It is made available under aCC-BY 4.0 International license.

48. Cagliero, C., Zhou, Y.N. and Jin, D.J. (2014) Spatial organization of transcription machinery and its segregation from the replisome in fast-growing bacterial cells. Nucleic Acids Research, 42, 13696-13705.

49. Mata Martin, C., Sun, Z., Zhou, Y.N. and Jin, D.J. (2018) Extrachromosomal Nucleolus-Like Compartmentalization by a Plasmid-Borne Ribosomal RNA Operon and Its Role in Nucleoid Compaction. Frontiers in Microbiology, 9, 1115.

50. Espejo, R.T. and Plaza, N. (2018) Multiple Ribosomal RNA Operons in Bacteria; Their Concerted Evolution and Potential Consequences on the Rate of Evolution of Their $16 \mathrm{~S}$ rRNA. Frontiers in Microbiology, 9, 1232.

51. Bratton Benjamin, P., Mooney Rachel, A. and Weisshaar James, C. (2011) Spatial Distribution and Diffusive Motion of RNA Polymerase in Live Escherichia coli. Journal of Bacteriology, 193, 5138-5146.

52. Jensen, K.F. and Pedersen, S. (1990) Metabolic growth rate control in Escherichia coli may be a consequence of subsaturation of the macromolecular biosynthetic apparatus with substrates and catalytic components. Microbiological Reviews, 54, 89-100.

53. Gummesson, B., Magnusson, L.U., Lovmar, M., Kvint, K., Persson, Ö., Ballesteros, M., Farewell, A. and Nyström, T. (2009) Increased RNA polymerase availability directs resources towards growth at the expense of maintenance. The EMBO Journal, 28, 2209-2219.

54. Slager, J., Kjos, M., Attaiech, L. and Veening, J.-W. (2014) Antibiotic-Induced Replication Stress Triggers Bacterial Competence by Increasing Gene Dosage near the Origin. Cell, 157, 395-406.

55. Merrikh, H., Machón, C., Grainger, W.H., Grossman, A.D. and Soultanas, P. (2011) Codirectional replication-transcription conflicts lead to replication restart. Nature, 470, 554-557. 